\title{
Second Order Impulsive Retarded Differential Inclusions with Nonlocal Conditions
}

\author{
Hernán R. Henríquez, ${ }^{1}$ Marcos Rabelo, ${ }^{2}$ and Luciana Vale ${ }^{2}$ \\ ${ }^{1}$ Departamento de Matemática, Universidad de Santiago (USACH), Casilla 307, Correo 2, Santiago, Chile \\ ${ }^{2}$ Departamento de Matemática, Universidade Federal de Goiás, Campus Catalão, 75704-020 Catalão, GO, Brazil \\ Correspondence should be addressed to Marcos Rabelo; rabelo@dmat.ufpe.br
}

Received 27 August 2013; Accepted 3 December 2013; Published 23 January 2014

Academic Editor: Geraldo Botelho

Copyright (c) 2014 Hernán R. Henríquez et al. This is an open access article distributed under the Creative Commons Attribution License, which permits unrestricted use, distribution, and reproduction in any medium, provided the original work is properly cited.

In this work we establish some existence results for abstract second order Cauchy problems modeled by a retarded differential inclusion involving nonlocal and impulsive conditions. Our results are obtained by using fixed point theory for the measure of noncompactness.

\section{Introduction}

In this paper we are interested in studying the existence of solutions to evolution systems that can be described by equations that suffer abrupt changes in their trajectories and simultaneously depend on nonlocal initial conditions. More specifically, the aim of this paper is to establish existence results for abstract second order evolution problems with delay whose equations can be written as differential inclusions with nonlocal initial conditions and subjected to impulses.

To describe the problem, throughout this work we denote by $X$ a Banach space provided with a norm $\|\cdot\|$. We assume that $A: D(A) \subseteq X \rightarrow X$ is the infinitesimal generator of a cosine functions of operators $C(t)$ on $X$. We study the system on an interval $J=[0, T]$, for some $T>0$, and we assume that the impulses occur at fixed moments $0<t_{1}<t_{2}, \ldots, t_{m}<T$. Moreover, $h>0$ denotes the system delay. Specifically, we will consider abstract second order systems

$$
\begin{gathered}
x^{\prime \prime}(t)-A x(t) \in F\left(t, x(t), x^{\prime}(t), x_{t}\right), \\
t \in J=[0, T], t \neq t_{k}, \quad k=1, \ldots, m, \\
\Delta x\left(t_{k}\right)=I_{k}^{1}\left(x\left(t_{k}\right), x^{\prime}\left(t_{k}\right), x_{t_{k}}\right), \quad k=1, \ldots, m, \\
\Delta x^{\prime}\left(t_{k}\right)=I_{k}^{2}\left(x\left(t_{k}\right), x^{\prime}\left(t_{k}\right), x_{t_{k}}\right), \quad k=1, \ldots, m,
\end{gathered}
$$

$$
\begin{gathered}
x(\theta)+g(x)(\theta)=\varphi(\theta), \quad \theta \in[-h, 0], \\
x^{\prime}(0)=z \in X,
\end{gathered}
$$

where $x(t) \in X, x_{t}, t \geq 0$ denotes the function defined by $x_{t}(\theta)=x(t+\theta)$ for $\theta \in[-h, 0], \Delta y(t)=y\left(t^{+}\right)-y\left(t^{-}\right)$indicates the gap of a piecewise continuous function $y(\cdot)$ at $t, \varphi$ : $[-h, 0] \rightarrow X$ is an appropriate function, and $F, I_{k}^{1}, I_{k}^{2}$, and $g$ are maps that will be specified later.

As a model, we consider a general wave equation described by a second order differential inclusion with impulses and nonlocal initial conditions

$$
\begin{gathered}
\frac{\partial^{2} u(t, \xi)}{\partial t^{2}}-\frac{\partial^{2} u(t, \xi)}{\partial \xi^{2}} \\
\epsilon \int_{0}^{\xi} f_{0}\left(t, \eta, u(t, \eta) \frac{\partial u(t, \eta)}{\partial t} \int_{-h}^{0} u(t+\theta, \eta) d \theta\right) d \eta \\
u(t, 0)=u(t, \pi)=0, \quad t \in[0, T] \\
u\left(t_{k}^{+}, \xi\right)=u\left(t_{k}, \xi\right)+a_{k}^{1}(\xi) \int_{0}^{\pi} q_{k}^{1}(\eta) u\left(t_{k}, \eta\right) d \eta+b_{k}^{1}(\xi)
\end{gathered}
$$




$$
\begin{aligned}
& \frac{\partial}{\partial t} u\left(t_{k}^{+}, \xi\right) \\
& \quad=\frac{\partial}{\partial t} u\left(t_{k}, \xi\right)+a_{k}^{2}(\xi) \int_{0}^{\pi} q_{k}^{2}(\eta) \frac{\partial}{\partial t} u\left(t_{k}, \eta\right) d \eta+b_{k}^{2}(\xi) \\
& u(\theta, \xi)+\sigma(\xi) \int_{0}^{T} \int_{0}^{\xi} u(t+\theta, \eta) d \eta d t \\
& \quad=\varphi(\theta, \xi), \quad \theta \in[-h, 0], \quad \frac{\partial}{\partial t} u(0, \xi)=z(\xi)
\end{aligned}
$$

for $t \in J=[0, T], \xi \in(0, \pi)$, and $k=1, \ldots, m$. In this system we assume that $f_{0}$ is a multivalued map, and the inclusion indicated in (5) will be explained in Section 4. Moreover, $a_{k}^{i}$, $b_{k}^{i}, q_{k}^{i}, i=1,2, \varphi$ and $z$ are appropriate functions.

Here we briefly discuss the context in which our work is inserted. We do not intend to make an exhaustive list of references but just mention those most recent and directly related to the topic of this paper. Differential inclusions and impulsive differential inclusions are used to describe many phenomena arising from different fields as physics, chemistry, population dynamics, and so forth. For this reason, last years several researchers have studied various aspects of the theory. We mention here to [1-6] and references in these texts for the motivations of the theory.

In particular, there are phenomena in nature that experiment abrupt changes at fixed moments of time. Such kind of systems are well described by impulsive systems. In the study of ordinary and partial differential equations with impulsive action, interesting questions appear such as local and global existence, stability, controllability, and so forth. For this reason this topic has attracted the attention of many authors in the last time. We only mention here the papers [7-17] which are directly related with the objective of this paper.

The concept of nonlocal initial condition was introduced by Byszewski and Lakshmikantham to extend the classical theory of initial value problems $([18-22])$. This notion is more appropriate than the classical theory to describe natural phenomena because it allows us to consider additional information. Thenceforth, the study of differential equations with nonlocal initial conditions has been an active topic of research. The interested reader can consult [23-26] and the references therein for recent developments on issues similar to those addressed in this paper.

On the other hand, it is well known that retarded functional differential equations are used to model important concrete phenomena. For general aspects of the theory of partial differential equations with delay we refer to [27], and for functional differential inclusions we refer to $[7,9,12-$ $14,28]$. In similar way, there exists an extensive literature concerning abstract second order problems. In the autonomous case, the existence of solutions to the second order abstract Cauchy problem is strongly related with the concept of cosine functions.

In this paper, we combine the theory of cosine functions with the properties of the measure of noncompactness and some properties of function spaces introduced in [9] to establish the existence of solutions to the problems (1)-(4).
This paper has four sections. In Section 2 we develop some properties about the abstract Cauchy problem of second order, the measure of noncompactness, and multivalued analysis which are needed to establish our results. In Section 3 we discuss the existence of mild solutions to problems (1)(4). Finally, in Section 4 we apply our results to establish the existence of solutions to problems (5)-(9).

The terminology and notations are those generally used in functional analysis. In particular, if $\left(Y,\|\cdot\|_{Y}\right)$ and $\left(Z,\|\cdot\|_{Z}\right)$ are Banach spaces, we denote by $\mathscr{L}(Y, Z)$ the Banach space of the bounded linear operators from $Y$ into $Z$ and we abbreviate this notation to $\mathscr{L}(Y)$ whenever $Z=Y$.

\section{Preliminaries}

2.1. The Second Order Abstract Cauchy Problem. In this section we collect the main facts concerning the existence of solutions for second order abstract differential equations. For the theory of cosine functions of operators we refer to [29$34]$. We next only mention a few concepts and properties relative to the second order abstract Cauchy problem. Throughout this paper, $A$ is the infinitesimal generator of a strongly continuous cosine function of bounded linear operators $C(t)$ on the Banach space $X$. We denote by $S(t)$ the sine function associated with $C(t)$ which is defined by

$$
S(t) x=\int_{0}^{t} C(s) x d s, \quad x \in X, t \in \mathbb{R} .
$$

We denote by $M, M_{1}$ some positive constants such that $\|C(t)\| \leq M$ and $\|S(t)\| \leq M_{1}$ for $t \in J$. The function $S: \mathbb{R} \rightarrow$ $\mathscr{L}(X)$ is continuous for the norm of operators and $\|S(t)\| \leq$ $M t$ for every $t \in J$. The notation $E$ stands for the space formed by the vectors $x \in X$ for which $C(\cdot) x$ is a function of class $C^{1}$ on $\mathbb{R}$. We know from Kisyński [35] that $E$ endowed with the norm

$$
\|x\|_{E}=\|x\|+\sup _{0 \leq t \leq 1}\|A S(t) x\|, \quad x \in E,
$$

is a Banach space.

The operator valued function $G(t)=\left[\begin{array}{cc}C(t) & S(t) \\ A S(t) & C(t)\end{array}\right]$ is a strongly continuous group of bounded linear operators on the space $E \times X$, generated by the operator $\mathscr{A}=\left[\begin{array}{ll}0 & I \\ A & 0\end{array}\right]$ defined on $D(A) \times E$. It follows from this property that $A S(t): E \rightarrow X$ is a bounded linear operator and that $A S(\cdot): \mathbb{R} \rightarrow \mathscr{L}(E ; X)$ is a strongly continuous operator valued map. We denote by $M_{2}$ a positive constant such that $\|A S(t)\|_{\mathscr{L}(E ; X)} \leq M_{2}$ for all $t \in J$. In addition ([34])

$$
S(t+s)=C(t) S(s)+C(s) S(t), \quad s, t \in \mathbb{R},
$$

which implies that

$$
\begin{array}{r}
A S(t+s) x=C(t) A S(s) x+C(s) A S(t) x, \\
x \in E, s, t \in \mathbb{R} .
\end{array}
$$

Furthermore, if $x:[0, \infty) \rightarrow X$ is a locally integrable function, then

$$
y(t)=\int_{0}^{t} S(t-s) x(s) d s
$$

defines an $E$-valued continuous function. 
The existence of solutions of the second order abstract Cauchy problem

$$
\begin{gathered}
x^{\prime \prime}(t)=A x(t)+f(t), \quad t \in J, \\
x(0)=z^{1}, \quad x^{\prime}(0)=z^{2},
\end{gathered}
$$

where $f:[0, T] \rightarrow X$ is an integrable function has been discussed in $[30,32-34,36]$. Similarly, the existence of solutions for the semilinear second order abstract Cauchy problem has been treated in [37]. We only mention here that the function $x(\cdot)$ given by

$$
x(t)=C(t) z^{1}+S(t) z^{2}+\int_{0}^{t} S(t-s) f(s) d s, \quad t \in J,
$$

is called mild solution of (15), and that when $z^{1} \in E$ the function $x(\cdot)$ is continuously differentiable and

$$
x^{\prime}(t)=A S(t) z^{1}+C(t) z^{2}+\int_{0}^{t} C(t-s) f(s) d s .
$$

2.2. Measure of Noncompactness and Multivalued Maps. In this subsection we recall some facts concerning multivalued analysis, which will be used later. Let $\Omega$ be a metric space. Throughout this paper $\mathscr{P}(\Omega)$ denotes the collection of all nonempty subsets of $\Omega$ and $\mathscr{P}_{b}(\Omega)$ denotes the collection of all bounded nonempty subsets of $\Omega$.

Some of our results are based on the concept of measure of noncompactness. For this reason, we next recall a few properties of this concept. For general information the reader can see $[5,9,38,39]$. In this paper, we use the notion of Hausdorff measure of noncompactness.

Definition 1. Let $B$ be a bounded subset of a metric space $\Omega$. The Hausdorff measure of noncompactness of $B$ is defined by

$$
\begin{aligned}
\eta(B)=\inf \{\varepsilon>0: B \text { has a finite cover } \\
\text { by closed balls of radius }<\varepsilon\} .
\end{aligned}
$$

Remark 2. Let $B_{1}, B_{2} \subseteq \Omega$ be bounded sets. The Hausdorff measure of noncompactness has the following properties.
(a) If $B_{1} \subseteq B_{2}$, then $\eta\left(B_{1}\right) \leqslant \eta\left(B_{2}\right)$.
(b) $\eta(B)=\eta(\bar{B})$.
(c) $\eta(B)=0$ if and only if $B$ is totally bounded.
(d) $\eta\left(B_{1} \cup B_{2}\right)=\max \left\{\eta\left(B_{1}\right), \eta\left(B_{2}\right)\right\}$.

In what follows, we assume that $Y$ is a normed space. For a bounded set $B \subseteq Y$, we denote by $\overline{c o}(B)$ the closed convex hull of the set $B$.

Remark 3. Let $B_{1}, B_{2} \subseteq Y$ be bounded sets. The following properties hold.
(a) For $\lambda \in \mathbb{R}, \eta(\lambda B)=|\lambda| \eta(B)$.
(b) $\eta\left(B_{1}+B_{2}\right) \leqslant \eta\left(B_{1}\right)+\eta\left(B_{2}\right)$, where $B_{1}+B_{2}=\left\{b_{1}+b_{2}\right.$ : $\left.b_{1} \in B_{1}, b_{2} \in B_{2}\right\}$.
(c) $\eta(B)=\eta(\overline{\mathrm{co}}(B))$.

Henceforth we use the notations $v(Y)$ and $\mathscr{K} v(Y)$ to denote the following sets:

(s1) $v(Y)=\{D \in \mathscr{P}(Y): D$ is convex $\}$,

(s2) $\mathscr{K} v(Y)=\{D \in v(Y): D$ is compact $\}$.

We refer the reader to the already mentioned references to abstract concepts of measure of noncompactness and for many examples of measure of noncompactness.

Definition 4. Let $\Omega$ be metric space. We said that a multivalued map $\mathscr{F}: \Omega \rightarrow \mathscr{P}(Y)$ is said to be

(i) upper semicontinuous (u.s.c. for short) if $\mathscr{F}^{-1}(V)=$ $\{w \in \Omega: \mathscr{F}(w) \subseteq V\}$ is an open subset of $\Omega$ for all open set $V \subseteq Y$;

(ii) closed if its graph $G_{\mathscr{F}}=\{(w, y) ; y \in \mathscr{F}(w)\}$ is a closed subset of $\Omega \times Y$;

(iii) compact if its range $\mathscr{F}(\Omega)$ is relatively compact in $Y$;

(iv) quasicompact if $\mathscr{F}(K)$ is relatively compact in $Y$ for any compact subset $K \subset \Omega$.

Definition 5. A multivalued map $\mathscr{F}: \Omega \rightarrow \mathscr{P}(Y)$ is said to be a condensing map with respect to $\eta$ (abbreviated, $\eta$ condensing) if for every bounded set $D \subset \Omega, \eta(D)>0$, $\eta(\mathscr{F}(D))<\eta(D)$.

The next result is essential for the development of the rest of our work. We point out that if $\mathscr{F}: \Omega \rightarrow K v(Y)$ is u.s.c., then $\mathscr{F}$ is closed. This allows us to establish the following version of the fixed point theorem [5, Corollary 3.3.1].

Theorem 6. Let $M$ be a convex closed subset of $Y$, and let $\mathscr{F}$ : $M \rightarrow K v(M)$ be a u.s.c. $\beta$-condensing multivalued map. Then Fix $(\mathscr{F})=\{y \in F(y)\}$ is a nonempty compact set.

2.3. Function Spaces. Let $I$ be any of the intervals $[0, T]$ or $[-h, T]$. The space $\mathrm{PC}(I ; X)$ is formed by all piecewise continuous functions $x: I \rightarrow X$ satisfying the following conditions:

(c1) the function $x(\cdot)$ is continuous on $I \backslash\left\{t_{1}, \ldots, t_{m}\right\}$, and

(c2) there exist $\lim _{t \rightarrow t_{j}^{+}} x(t)$ and $\lim _{t \rightarrow t_{j}^{-}}$and $x\left(t_{j}\right)=$ $\lim _{t \rightarrow t_{j}^{-}} x(t)$ for all $1 \leq j \leq m$.

We consider PC $(I ; X)$ endowed with the norm of the uniform convergence

$$
\|x\|_{\mathrm{PC}}=\sup _{t \in I}\|x(t)\| .
$$

It is well known that $\mathrm{PC}(I ; X)$ is a Banach space. Furthermore, let $\Pi_{j}: \operatorname{PC}(I ; X) \rightarrow C\left(\left[t_{j}, t_{j+1}\right] ; X\right),-1 \leq j \leq m$, be the map defined by

$$
\Pi_{j}(x)(t)= \begin{cases}x(t) & \text { if } t \in\left(t_{j}, t_{j+1}\right] \\ x\left(t_{j}^{+}\right) & \text {if } t=t_{j}\end{cases}
$$

where we set $t_{-1}=-h, t_{0}=0$, and $t_{m+1}=T$. For each $j \epsilon$ $\{0, \ldots, m\}$ and $D \subseteq \mathrm{PC}([-h, T] ; X)$, we denote by $D_{j}$ the range of $D$ under the operator $\Pi_{j}$; that is, $D_{j}=\Pi_{j}(D)$. 
We define the subspace $\mathrm{PC}^{1}([-h, T] ; X)$ of $\mathrm{PC}([-h, T] ; X)$ consisting of all functions which are continuously differentiable at $[0, T] \backslash\left\{t_{1}, \ldots, t_{m}\right\}$ and there exist $x^{\prime}\left(t_{k}^{+}\right)$and $x^{\prime}\left(t_{k}^{-}\right)$ for all $1 \leq k \leq m$. It is straightforward to show that the space $\mathrm{PC}^{1}([-h, T] ; X)$ endowed with the norm

$$
\|x\|_{\mathrm{PC}^{1}}=\|x\|_{\mathrm{PC}}+\sup _{0 \leq t \leq T}\left\|x^{\prime}(t)\right\|
$$

is a Banach space.

From now on we denote by $C_{h}^{p}, 1 \leq p<\infty$, the space of piecewise continuous functions $v:[-h, 0] \rightarrow X$ endowed with the norm

$$
\|v\|_{C_{h}^{p}}=\frac{1}{h^{1 / p}}\left(\int_{-h}^{0}\|v(\theta)\|^{p} d \theta\right)^{1 / p} .
$$

In what follows we denote by $\chi$ the Hausdorff measure of noncompactness in $X$ and by $\beta$ the Hausdorff measure of noncompactness in a space of continuous (or piecewise continuous) functions with values in $X$. We next collect some properties of measure $\beta$ which are needed to establish our results.

Lemma 7. Let $G:[0, T] \rightarrow \mathscr{L}(X)$ be a strongly continuous operator valued map. Let $D \subset X$ be a bounded set. Then $\beta(\{G(\cdot) x: x \in D\}) \leq \sup _{0 \leq t \leq T}\|G(t)\| \chi(D)$.

Lemma 8. Let $D \subset P C([-h, T] ; X)$ be a bounded set. Then $\beta(D)=\max _{k=-1, \ldots, m} \beta\left(D_{k}\right)$.

Lemma 9 (see [39]). Let $W \subseteq C(J ; X)$ be a bounded set. Then $\chi(W(t)) \leqslant \beta(W)$ for all $t \in J$. Furthermore, if $W$ is equicontinuous on $J$, then $\chi(W(t))$ is continuous on $J$, and

$$
\beta(W)=\sup \{\chi(W(t)): t \in J\} .
$$

Lemma 10. Let $D \subseteq C(J ; X)$ be a bounded set. Then there exists a countable set $D_{0} \subseteq D$ such that $\beta\left(D_{0}\right)=\beta(D)$.

A set $W \subseteq L^{1}(J ; X)$ is said to be uniformly integrable if there exists a positive function $\mu \in L^{1}(J)$ such that $\|w(t)\| \leq$ $\mu(t)$ a.e. for $t \in J$ and all $w \in W$.

Lemma 11. Let $G:[0, T] \rightarrow \mathscr{L}(X)$ be a strongly continuous operator valued map and $\Lambda: L^{1}([0, T] ; X) \rightarrow C([0, T] ; X)$ be the map defined by

$$
\Lambda(u)(t)=\int_{0}^{t} G(t-s) u(s) d s .
$$

Let $W \subset L^{1}([0, T] ; X)$. Assume that there is a compact set $K \subset$ $X$ and a positive function $q \in L^{1}([0, T])$ such that $W(t) \subseteq K$ for all $t \in[0, T]$ and $\chi(W(t)) \leq q(t)$. Then

$$
\beta(\Lambda(W)) \leq 2 \sup _{0 \leq t \leq T}\|G(t)\| \int_{0}^{T} q(t) d t .
$$

Proof. It is clear that $W$ is uniformly integrable. Applying Lemma 10 and [5, Theorem 4.2.2], we can affirm that

$$
\chi(\Lambda(W)(t)) \leq 2 \sup _{0 \leq t \leq T}\|G(t)\| \int_{0}^{t} q(s) d s .
$$

Since the set $\Lambda(W)$ is equicontinuous, using Lemma 11, we obtain the assertion.

We also need to consider the product space $\mathrm{PC}([-h, T]$; $X) \times \mathrm{PC}([0, T] ; X)$ provided with the norm

$$
\|(x, y)\| \|=\max \left\{\|x\|_{\mathrm{PC}([-h, T] ; X)},\|y\|_{\mathrm{PC}([0, T] ; X)}\right\} .
$$

The following property is immediate.

Lemma 12. Let $W \subset P C([-h, T] ; X) \times P C([0, T] ; X)$ be a bounded set.

(a) Assume that $W=W_{1} \times W_{2}$, where $W_{1} \subset P C([-h, T]$; $X)$ and $W_{2} \subset P C([0, T] ; X)$ are bounded sets. Then $\beta(W)=\max \left\{\beta\left(W_{1}\right), \beta\left(W_{2}\right)\right\}$.

(b) Let

$$
\begin{array}{r}
W_{1}=\{x \in P C([-h, T] ; X):(x, y) \in W, \\
\text { for some } y \in P C([0, T] ; X)\}, \\
W_{2}=\{y \in P C([0, T] ; X):(x, y) \in W, \\
\text { for some } x \in P C([-h, T] ; X)\} .
\end{array}
$$

Then $\max \left\{\beta\left(W_{1}\right), \beta\left(W_{2}\right)\right\} \leq \beta(W)$.

\section{Existence Results}

In this section we establish some results of existence of mild solutions of problems (1)-(4). Initially we will establish the general framework of conditions under which we will study this problem. Throughout this section, $\chi$ denotes the Hausdorff measure of noncompactness in $X$. We assume that $\varphi \in C([-h, 0] ; X)$. Moreover, in what follows we assume that $F$ is a multivalued map from $J \times X \times X \times C_{h}^{p}$ into $\mathscr{K} v(X)$ that satisfies the following properties.

(F1) The function $F\left(\cdot, y^{1}, y^{2}, \psi\right):[0, T] \rightarrow \mathscr{K} v(X)$ admits a strongly measurable selection for each $y^{i} \in$ $X, i=1,2$, and $\psi \in C_{h}^{p}$.

(F2) For each $t \in[0, T]$, the function $F(t, \cdot, \cdot, \cdot): X \times X \times$ $C_{h}^{p} \rightarrow \mathscr{K} v(X)$ is u.s.c.

(F3) For each $r>0$, there is a function $\mu_{r} \in L^{1}([0, T])$ such that

$\left\|F\left(t, y^{1}, y^{2}, \psi\right)\right\|:=\sup \left\{\|v\|: v \in F\left(t, y^{1}, y^{2}, \psi\right)\right\} \leq \mu_{r}(t)$, a.e. $t \in[0, T]$,

for all $y^{i} \in X, i=1,2$, and $\psi \in C_{h}^{p}$ such that

$$
\left\|y^{1}\right\|+\left\|y^{2}\right\|+\|\psi\|_{C_{h}^{p}} \leq r
$$


(F4) There exists a positive integrable function $k(\cdot)$ on $[0, T]$ such that

$$
\begin{aligned}
\chi\left(F\left(t, \Omega_{1}, \Omega_{2}, Q\right)\right) \leq & k(t)\left(\chi\left(\Omega_{1}\right)+\chi\left(\Omega_{2}\right)\right) \\
& +\sup _{\theta \in[-h, 0]} \chi(Q(\theta)), \quad \text { a.e. } t \in[0, T],
\end{aligned}
$$

for all bounded sets $\Omega_{i} \subseteq X, i=1,2$, and $Q \subseteq C_{h}^{p}$ such that $\sup _{\theta \in[-h, 0]}\{\|\psi(\theta)\|: \psi \in Q\}<\infty$.

Remark 13. Let $x(\cdot) \in \mathrm{PC}([-h, T] ; X)$ and $y(\cdot) \in \mathrm{PC}([0$, $T] ; X)$. Then the function $[0, T] \rightarrow C_{h}^{p}, t \mapsto x_{t}$, is continuous. Hence, the function $[0, T] \rightarrow X \times X \times C_{h}^{p}, t \mapsto(x(t)$, $\left.y(t), x_{t}\right)$, is strongly measurable. Combining this assertion with conditions (F1) and (F2) and applying [5, Theorem 1.3.5] we infer that the function $[0, T] \rightarrow \mathscr{K} v(X), t \mapsto$ $F\left(t, x(t), y(t), x_{t}\right)$ admits a Bochner integrable selection. As a consequence, the set

$$
\begin{aligned}
& \mathcal{S}_{F, x, y} \\
& \quad=\left\{f \in L^{1}(J ; X): f(t) \in F\left(t, x(t), y(t), x_{t}\right), t \in J\right\} \neq \emptyset,
\end{aligned}
$$

and $\mathcal{S}_{F, x, y}$ is convex.

Next we introduce the conditions on the function $g$. We assume that $g$ is a map from $\mathrm{PC}([-h, T] ; X)$ into $C([-h, 0] ; X)$ such that the values $g(x)(0) \in E$ for all $x(\cdot) \in \mathrm{PC}([-h, T] ; X)$ and that the following conditions are fulfilled.

(g1) The function $g$ is continuous and takes bounded sets in $\mathrm{PC}([-h, T] ; X)$ into bounded subsets of $C([-h, 0] ; X)$. Moreover, the map $g(\cdot)(0): \operatorname{PC}([-h$, $T] ; X) \rightarrow E$ is continuous and takes bounded sets in $\mathrm{PC}([-h, T] ; X)$ into bounded subsets of $E$.

(g2) There is a continuous function $\ell:[-h, 0] \rightarrow[0, \infty)$ and a constant $\ell_{1} \geq 0$ such that

$$
\begin{gathered}
\chi(g(W)(\theta)) \leq \ell(\theta) \sup _{t \in[-h, T]} \chi(W(t)), \quad \theta \in[-h, 0] \\
\chi_{E}(g(W)(0)) \leq \ell_{1} \sup _{t \in[-h, T]} \chi(W(t))
\end{gathered}
$$

for all bounded set $W \subset \mathrm{PC}([-h, T], X)$.

(g3) For each bounded set $W \subset \mathrm{PC}([-h, T] ; X)$ the set $g(W)$ is equicontinuous.

Next we establish the conditions on maps $I_{k}^{i}, i=1,2, k=$ $1, \ldots, m$.

We assume that $I_{k}^{1}: X \times X \times C_{h}^{p} \rightarrow E$ and $I_{k}^{2}: X \times X \times$ $C_{h}^{p} \rightarrow X$ satisfy the following conditions.

(I1) The maps $I_{k}^{i}, i=1,2, k=1, \ldots, m$ are continuous and takes bounded sets into bounded sets.
(I2) There are positive constants $d_{k}^{i, j}, i=1,2, j=0,1,2$, $k=1, \ldots, m$, such that

$$
\begin{aligned}
& \chi_{E}\left(I_{k}^{1}\left(D_{0} \times D_{1} \times W\right)\right) \\
& \quad \leq d_{k}^{1,0} \chi\left(D_{0}\right)+d_{k}^{1,1} \chi\left(D_{1}\right)+d_{k}^{1,2} \sup _{-h \leq \theta \leq 0} \chi(W(\theta)), \\
& \chi\left(I_{k}^{2}\left(D_{0} \times D_{1} \times W\right)\right) \\
& \quad \leq d_{k}^{2,0} \chi\left(D_{0}\right)+d_{k}^{2,1} \chi\left(D_{1}\right)+d_{k}^{2,2} \sup _{-h \leq \theta \leq 0} \chi(W(\theta)),
\end{aligned}
$$

for all bounded subsets $D_{0}, D_{1}$ of $X$, and $W \subseteq C_{h}^{p}$ such that $\sup _{\theta \in[-h, 0]}\{\|\psi(\theta)\|: \psi \in W\}<\infty$.

Remark 14. Let $W \subset \mathrm{PC}([-h, T] ; X)$ be a bounded set. Then for all $t \in[0, T], W_{t}=\left\{w_{t}: w \in W\right\}$ is a bounded subset of $C_{h}^{p}$ and $\sup _{-h \leq \theta \leq 0} \chi\left(W_{t}(\theta)\right) \leq \beta(W)$ for all $0 \leq t \leq T$.

Motivated by expressions (16) and (17) (see also [12]), we introduce the following concept of mild solution to problems (1)-(4).

Definition 15. A function $x(\cdot) \in \mathrm{PC}^{1}([-h, T] ; X)$ is said to be a mild solution of (1)-(4) if conditions (2)-(4) are satisfied, and the integral equation

$$
\begin{aligned}
x(t)= & C(t)(\varphi(0)-g(x)(0))+S(t) z \\
& +\int_{0}^{t} S(t-s) f(s) d s \\
& +\sum_{t_{k}<t} C\left(t-t_{k}\right) I_{k}^{1}\left(x\left(t_{k}\right), x^{\prime}\left(t_{k}\right), x\left(t_{k}\right)\right) \\
& +\sum_{t_{k}<t} S\left(t-t_{k}\right) I_{k}^{2}\left(x\left(t_{k}\right), x^{\prime}\left(t_{k}\right), x\left(t_{k}\right)\right),
\end{aligned}
$$

is verified for $f \in \mathcal{S}_{F, x, x^{\prime}}$ and all $t \in[0, T]$.

To establish our results, we need to study two integral operators defined on the set $\mathcal{S}_{F, x, y}$ for functions $x \in$ $\operatorname{PC}([-h, T] ; X)$ and $y \in \operatorname{PC}([0, T] ; X)$. Initially we mention some properties of $\mathcal{S}_{F, x, y}$. A first result establishes that $\mathcal{S}_{F, x, y}$ is closed. Specifically we have the following property ([5, Lemma 5.1.1]).

Lemma 16. Let $\left\{x_{n}\right\}_{n=1}^{\infty} \subset P C([-h, T] ; X)$ and $\left\{y_{n}\right\}_{n=1}^{\infty} \subset$ $P C([0, T] ; X)$ be sequences that converge to $x_{0} \in P C([-h$, $T] ; X)$ and $y_{0} \in P C([0, T] ; X)$, respectively. Suppose that $\left\{f_{n}\right\}_{n=1}^{\infty} \subset L^{1}([0, T] ; X), f_{n} \in \mathcal{S}_{F, x_{n}, y_{n}}$, is a sequence that converges weakly to $f_{0} \in L^{1}([0, T] ; X)$. Then $f_{0} \in \mathcal{S}_{F, x_{0}, y_{0}}$.

On the other hand, since the values of $F$ are convex compact sets, and, as already mentioned, the graph of $F$ is closed, we can assert that for functions $x(\cdot) \in \mathrm{PC}([-h, T] ; X)$ and $y(\cdot) \in \operatorname{PC}([0, T] ; X)$, the set $\cup_{0 \leq t \leq t} F\left(t, x(t), y(t), x_{t}\right)$ is compact in $X$. In addition, as a consequence of (F3), the set $\mathcal{S}_{F, x, y}$ is uniformly integrable over $J$; that is to say, there exists 
a positive function $\mu_{x, y} \in L^{1}(J)$ such that $\|f(t)\| \leq \mu_{x, y}(t)$ a.e. for $t \in J$ and all $f \in \mathcal{S}_{F, x, y}$.

We introduce now the operators $\Lambda_{1}, \Lambda_{2}: L^{1}([0, T]$; $X) \rightarrow C([0, T] ; X)$ given by

$$
\begin{aligned}
& \Lambda_{1} f(t)=\int_{0}^{t} S(t-s) f(s) d s, \\
& \Lambda_{2} f(t)=\int_{0}^{t} C(t-s) f(s) d s .
\end{aligned}
$$

It is clear that $\Lambda_{1}, \Lambda_{2}$ are bounded linear operators. Using $\Lambda_{1}, \Lambda_{2}$ we can construct the multivalued maps $\widetilde{\Lambda}_{1}, \widetilde{\Lambda}_{2}$ : $\mathrm{PC}([-h, T] ; X) \times \mathrm{PC}([0, T] ; X) \rightarrow v(C([0, T] ; X))$ given by

$$
\begin{aligned}
& \widetilde{\Lambda}_{1}(x, y)=\Lambda_{1}\left(\mathcal{S}_{F, x, y}\right), \\
& \widetilde{\Lambda}_{2}(x, y)=\Lambda_{2}\left(\mathcal{S}_{F, x, y}\right) .
\end{aligned}
$$

Since $C(\cdot)$ and $S(\cdot)$ are strongly continuous operator valued functions, the assertion in [5, Lemma 4.2.1] remains valid for $\Lambda_{1}$ and $\Lambda_{2}$. Hence, combining our previous remarks with [5, Lemma 4.2.1, Corollary 5.1.2] we can establish the following property.

Lemma 17. Let $F:[0, T] \times X \times X \times C_{h}^{p} \rightarrow \mathscr{K} v(X)$ be a multivalued map satisfying conditions (F1)-(F4). Then $\widetilde{\Lambda}_{1}$ and $\widetilde{\Lambda}_{2}$ are U.s.c. maps with convex compact values.

We next define the solution map for problems (1)-(4) as follows. Assume that $\varphi(0) \in E$ and let $x \in \mathrm{PC}^{1}([-h, T] ; X)$. We define $\Gamma(x)$ to be the set formed by all functions $u$ given by

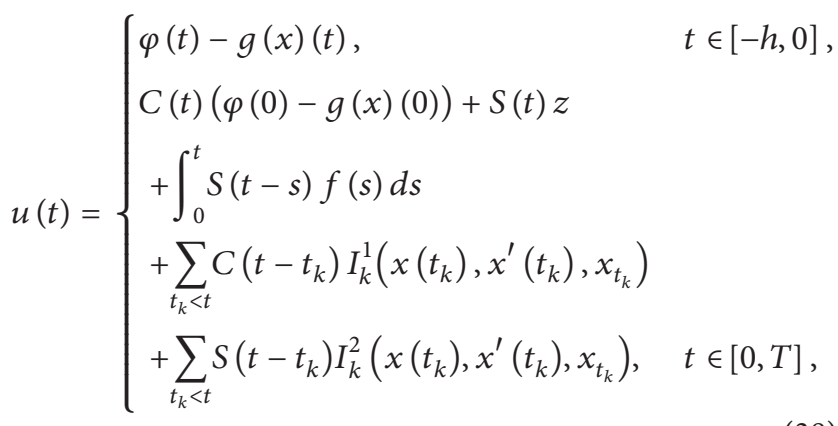

for $f \in \mathcal{S}_{F, x, x^{\prime}}$. It follows from our hypotheses that $u \in \operatorname{PC}^{1}([-h, T] ; X)$. Hence, $\Gamma \quad: \operatorname{PC}^{1}([-h, T] ; X) \rightarrow$ $\mathscr{P}\left(\mathrm{PC}^{1}([-h, T] ; X)\right)$. Furthermore, it is clear that $x(\cdot)$ is a mild solution of problems (1)-(4) if and only if $x(\cdot)$ is a fixed point of $\Gamma$.

We are now in a position to prove the main result of this section. We introduce the map $\mathscr{F}: \operatorname{PC}([-h, T] ; X) \times$ $\mathrm{PC}([0, T] ; X) \rightarrow \mathscr{P}(\mathrm{PC}([-h, T] ; X) \times \mathrm{PC}([0, T] ; X))$ defined as follows. For $(x, y) \in \mathrm{PC}([-h, T] ; X) \times \mathrm{PC}([0, T] ; X)$, $\mathscr{F}(x, y)$ is the set consisting of all functions $(u, v)$ given by

$u(t)$

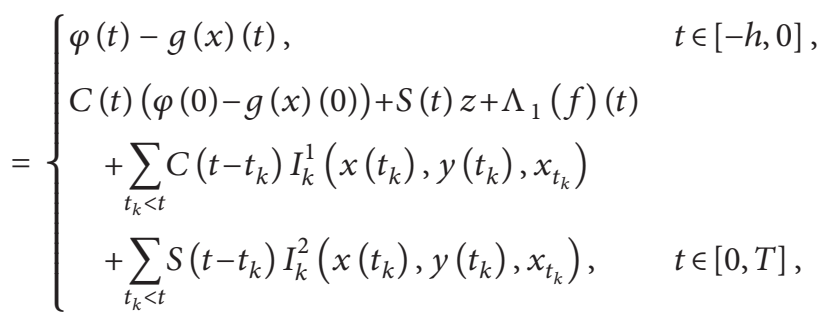

$v(t)$

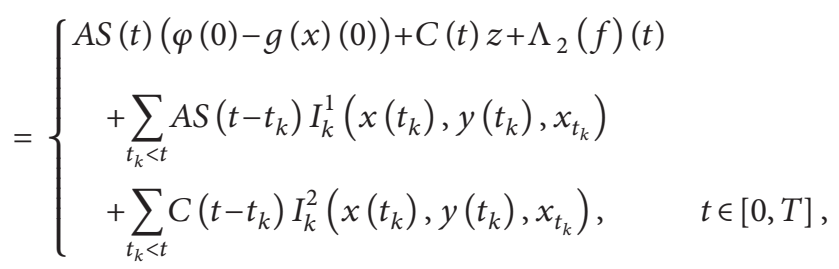

for $f \in \mathcal{S}_{F, x, y}$. It follows from (g1) and (I1) that $\mathscr{F}$ is well defined.

We use the following notations:

$$
\begin{gathered}
N_{i}=\sum_{k=1}^{m}\left(d_{k}^{i, 0}+d_{k}^{i, 2}\right), \quad i=1,2, \\
N_{i+2}=\sum_{k=1}^{m} d_{k}^{i, 1}, \quad i=1,2, \\
N_{5}=\max \left\{\max _{-h \leq \theta \leq 0} \ell(\theta), M \ell(0)+6 M_{1} \int_{0}^{T} k(t) d t\right. \\
\left.+M\left(N_{1}+N_{3}\right)+M_{1}\left(N_{2}+N_{4}\right)\right\}, \\
N_{6}=M_{2} \ell_{1} \\
+6 M \int_{0}^{T} k(t) d t+M_{2}\left(N_{1}+N_{3}\right)+M\left(N_{2}+N_{4}\right), \\
N_{7}=\max \left\{N_{5}, N_{6}\right\} .
\end{gathered}
$$

Theorem 18. Assume that $\varphi(0) \in E$, and conditions (F1)-(F4), (g1)-(g3) and (I1)-(I2) are fulfilled. If $N_{7}<1$, then the map $\mathscr{F}: P C([-h, T] ; X) \times P C([0, T] ; X) \rightarrow K v(P C([-h, T] ; X) \times$ $P C([0, T] ; X))$ is u.s.c. and $\beta$-condensing.

Proof. It follows from our hypotheses and Lemma 17 that $\mathscr{F}$ is a u.s.c. multivalued map with convex compact values. It remains to prove that $\mathscr{F}$ is $\beta$-condensing. Let $\Omega \subset \operatorname{PC}([-h, T] ; X) \times \operatorname{PC}([0, T] ; X)$ be a bounded set such that $\beta(\mathscr{F}(\Omega)) \geq \beta(\Omega)$. It follows from Lemma 10 that there exists a sequence $\left(w_{n}\right)_{n}$ in $\mathscr{F}(\Omega)$ such that 
$\beta(\mathscr{F}(\Omega))=\beta\left(\left\{w_{n}: n \in \mathbb{N}\right\}\right)$. We can write $w_{n}=\left(u_{n}, v_{n}\right) \epsilon$ $\mathscr{F}\left(x_{n}, y_{n}\right)$ for some $\left(x_{n}, y_{n}\right) \in \Omega$. It follows from Lemma 12 that

$\beta\left(\left\{w_{n}: n \in \mathbb{N}\right\}\right) \leq \max \left\{\beta\left(\left\{u_{n}: n \in \mathbb{N}\right\}\right), \beta\left(\left\{v_{n}: n \in \mathbb{N}\right\}\right)\right\}$.

Here we will estimate separately the values $\beta\left(\left\{u_{n}: n \in \mathbb{N}\right\}\right)$ and $\beta\left(\left\{v_{n}: n \in \mathbb{N}\right\}\right)$. To estimate $\beta\left(\left\{u_{n}: n \in \mathbb{N}\right\}\right)$, using (39), we can write

$$
\begin{aligned}
& u_{n}(t) \\
& = \begin{cases}\varphi(t)-g\left(x_{n}\right)(t), & t \in[-h, 0], \\
C(t)\left(\varphi(0)-g\left(x_{n}\right)(0)\right)+S(t) z+\Lambda_{1}\left(f_{n}\right)(t) & \\
+\sum_{t_{k}<t} C\left(t-t_{k}\right) I_{k}^{1}\left(x_{n}\left(t_{k}\right), y_{n}\left(t_{k}\right),\left(x_{n}\right)_{t_{k}}\right) & \\
+\sum_{t_{k}<t} S\left(t-t_{k}\right) I_{k}^{2}\left(x_{n}\left(t_{k}\right), y_{n}\left(t_{k}\right),\left(x_{n}\right)_{t_{k}}\right), & t \in[0, T],\end{cases}
\end{aligned}
$$

for $f_{n} \in \mathcal{S}_{F, x_{n}, y_{n}}$.

For $\theta \in[-h, 0]$, applying (g2), we get

$$
\begin{aligned}
& \chi\left(\left\{u_{n}(\theta): n \in \mathbb{N}\right\}\right)=\chi\left(\left\{g\left(x_{n}\right)(\theta): n \in \mathbb{N}\right\}\right) \\
& \leq \ell(\theta) \sup _{t \in[-h, T]} \chi\left(\left\{x_{n}(t): n \in \mathbb{N}\right\}\right) .
\end{aligned}
$$

Using now condition (g3) and Lemma 9 we infer that

$$
\beta\left(\left\{\varphi-g\left(x_{n}\right): n \in \mathbb{N}\right\}\right) \leq \max _{-h \leq \theta \leq 0} \ell(\theta) \beta\left(\left\{x_{n}: n \in \mathbb{N}\right\}\right) .
$$

Now we consider functions $u_{n}$ defined on $[0, T]$. From (43) and using Lemma 7, we get

$$
\begin{aligned}
& \beta\left(\left\{u_{n}(\cdot): n \in \mathbb{N}\right\}\right) \\
& \leq M \chi\left(\left\{g\left(x_{n}\right)(0): n \in \mathbb{N}\right\}\right)+\beta\left(\left\{\Lambda_{1}\left(f_{n}\right)(\cdot): n \in \mathbb{N}\right\}\right) \\
& \quad+M \sum_{k=1}^{m} \chi\left(\left\{I_{k}^{1}\left(x_{n}\left(t_{k}\right), y_{n}\left(t_{k}\right),\left(x_{n}\right)_{t_{k}}\right): n \in \mathbb{N}\right\}\right) \\
& \quad+M_{1} \sum_{k=1}^{m} \chi\left(\left\{I_{k}^{2}\left(x_{n}\left(t_{k}\right), y_{n}\left(t_{k}\right),\left(x_{n}\right)_{t_{k}}\right): n \in \mathbb{N}\right\}\right) .
\end{aligned}
$$

Using now conditions (g2), (I2), and Remark 14, we have

$$
\begin{aligned}
& \chi(\left.\left\{g\left(x_{n}\right)(0): n \in \mathbb{N}\right\}\right) \leq \ell(0) \beta\left(\left\{x_{n}: n \in \mathbb{N}\right\}\right), \\
& \chi(\{\left.\left.I_{k}^{i}\left(x_{n}\left(t_{k}\right), y_{n}\left(t_{k}\right),\left(x_{n}\right)_{t_{k}}\right): n \in \mathbb{N}\right\}\right) \\
& \quad \leq d_{k}^{i, 0} \chi\left(\left\{x_{n}\left(t_{k}\right): n \in \mathbb{N}\right\}\right)+d_{k}^{i, 1} \chi\left(\left\{y_{n}\left(t_{k}\right): n \in \mathbb{N}\right\}\right) \\
& \quad+d_{k}^{i, 2} \beta\left(\left\{x_{n}: n \in \mathbb{N}\right\}\right) \\
& \quad \leq\left(d_{k}^{i, 0}+d_{k}^{i, 2}\right) \beta\left(\left\{x_{n}: n \in \mathbb{N}\right\}\right)+d_{k}^{i, 1} \beta\left(\left\{y_{n}: n \in \mathbb{N}\right\}\right) .
\end{aligned}
$$

On the other hand, since $f_{n} \in \mathcal{S}_{F, x_{n}, y_{n}}$, for $t \in[0, T]$ we have that $f_{n}(t) \in F\left(t, x_{n}(t), y_{n}(t),\left(x_{n}\right)_{t}\right)$. This implies that $\left\{f_{n}: n \in\right.$ $\mathbb{N}\}$ is uniformly integrable and, applying condition (F4),

$$
\begin{aligned}
& \chi\left(\left\{f_{n}(t): n \in \mathbb{N}\right\}\right) \\
& \leq k(t)\left[\chi\left(\left\{x_{n}(t): n \in \mathbb{N}\right\}\right)+\chi\left(\left\{y_{n}(t): n \in \mathbb{N}\right\}\right)\right. \\
& \left.+\sup _{-h \leq \theta \leq 0} \chi\left(\left\{x_{n}(t+\theta): n \in \mathbb{N}\right\}\right)\right] \\
& \leq k(t)\left[2 \beta\left(\left\{x_{n}: n \in \mathbb{N}\right\}\right)+\beta\left(\left\{y_{n}: n \in \mathbb{N}\right\}\right)\right] . \\
& \beta\left(\left\{\Lambda_{1}\left(f_{n}\right)(\cdot): n \in \mathbb{N}\right\}\right) \\
& \leq 2 M_{1}\left[2 \beta\left(\left\{x_{n}: n \in \mathbb{N}\right\}\right)+\beta\left(\left\{y_{n}: n \in \mathbb{N}\right\}\right)\right] \int_{0}^{T} k(t) d t .
\end{aligned}
$$

Substituting in (46), we obtain

$$
\begin{aligned}
& \beta\left(\left\{u_{n}(\cdot): n \in \mathbb{N}\right\}\right) \\
& \leq M \ell(0) \beta\left(\left\{x_{n}: n \in \mathbb{N}\right\}\right) \\
& +2 M_{1}\left[2 \beta\left(\left\{x_{n}: n \in \mathbb{N}\right\}\right)\right. \\
& \left.+\beta\left(\left\{y_{n}: n \in \mathbb{N}\right\}\right)\right] \int_{0}^{T} k(t) d t \\
& +M \sum_{k=1}^{m}\left[\left(d_{k}^{1,0}+d_{k}^{1,2}\right) \beta\left(\left\{x_{n}: n \in \mathbb{N}\right\}\right)\right. \\
& \left.+d_{k}^{1,1} \beta\left(\left\{y_{n}: n \in \mathbb{N}\right\}\right)\right] \\
& +M_{1} \sum_{k=1}^{m}\left[\left(d_{k}^{2,0}+d_{k}^{2,2}\right) \beta\left(\left\{x_{n}: n \in \mathbb{N}\right\}\right)\right. \\
& \left.+d_{k}^{2,1} \beta\left(\left\{y_{n}: n \in \mathbb{N}\right\}\right)\right] \\
& \leq\left[M \ell(0)+4 M_{1} \int_{0}^{T} k(t) d t+M N_{1}+M_{1} N_{2}\right] \\
& \times \beta\left(\left\{x_{n}: n \in \mathbb{N}\right\}\right) \\
& +\left[2 M_{1} \int_{0}^{T} k(t) d t+M N_{3}+M_{1} N_{4}\right] \\
& \times \beta\left(\left\{y_{n}: n \in \mathbb{N}\right\}\right) \\
& \leq\left[M \ell(0)+6 M_{1} \int_{0}^{T} k(t) d t+M\left(N_{1}+N_{3}\right)\right. \\
& \left.+M_{1}\left(N_{2}+N_{4}\right)\right] \beta\left(\left\{\left(x_{n}, y_{n}\right): n \in \mathbb{N}\right\}\right) .
\end{aligned}
$$


Combining with (45), and using Lemma 12, it yields

$$
\begin{aligned}
& \beta\left(\left\{u_{n}(\cdot): n \in \mathbb{N}\right\}\right) \\
& \leq \max \left\{\max _{-h \leq \theta \leq 0} \ell(\theta) \beta\left(\left\{x_{n}: n \in \mathbb{N}\right\}\right),\right. \\
& \quad\left[M \ell(0)+6 M_{1} \int_{0}^{T} k(t) d t+M\left(N_{1}+N_{3}\right)\right. \\
& \left.\left.\quad+M_{1}\left(N_{2}+N_{4}\right)\right] \beta\left(\left\{\left(x_{n}, y_{n}\right): n \in \mathbb{N}\right\}\right)\right\} \\
& \leq N_{5} \beta\left(\left\{\left(x_{n}, y_{n}\right): n \in \mathbb{N}\right\}\right) .
\end{aligned}
$$

We next estimate $\beta\left(\left\{v_{n}: n \in \mathbb{N}\right\}\right)$. Using (40) we can write

$$
\begin{aligned}
& v_{n}(t) \\
& =\left\{\begin{array}{l}
A S(t)\left(\varphi(0)-g\left(x_{n}\right)(0)\right)+C(t) z+\Lambda_{2}\left(f_{n}\right)(t) \\
+\sum_{t_{k}<t} A S\left(t-t_{k}\right) I_{k}^{1}\left(x_{n}\left(t_{k}\right), y_{n}\left(t_{k}\right),\left(x_{n}\right)_{t_{k}}\right) \\
+\sum_{t_{k}<t} C\left(t-t_{k}\right) I_{k}^{2}\left(x_{n}\left(t_{k}\right), y_{n}\left(t_{k}\right),\left(x_{n}\right)_{t_{k}}\right), \quad t[0, T],
\end{array}\right.
\end{aligned}
$$

for $f_{n} \in \mathcal{S}_{F, x_{n}, y_{n}}$.

From (52) and using Lemma 7, we get

$$
\begin{aligned}
& \beta\left(\left\{v_{n}(\cdot): n \in \mathbb{N}\right\}\right) \\
& \leq M_{2} \chi_{E}\left(\left\{g\left(x_{n}\right)(0): n \in \mathbb{N}\right\}\right)+\beta\left(\left\{\Lambda_{2}\left(f_{n}\right)(\cdot): n \in \mathbb{N}\right\}\right) \\
& \quad+M_{2} \sum_{k=1}^{m} \chi_{E}\left(\left\{I_{k}^{1}\left(x_{n}\left(t_{k}\right), y_{n}\left(t_{k}\right),\left(x_{n}\right)_{t_{k}}\right): n \in \mathbb{N}\right\}\right) \\
& \quad+M \sum_{k=1}^{m} \chi\left(\left\{I_{k}^{2}\left(x_{n}\left(t_{k}\right), y_{n}\left(t_{k}\right),\left(x_{n}\right)_{t_{k}}\right): n \in \mathbb{N}\right\}\right) .
\end{aligned}
$$

Using again conditions (g2) and (I2), Lemma 12, and also our previous estimates, we obtain

$$
\begin{aligned}
& \beta\left(\left\{v_{n}(\cdot): n \in \mathbb{N}\right\}\right) \\
& \leq M_{2} \ell_{1} \beta\left(\left\{x_{n}: n \in \mathbb{N}\right\}\right) \\
& \quad+2 M\left[2 \beta\left(\left\{x_{n}: n \in \mathbb{N}\right\}\right)+\beta\left(\left\{y_{n}: n \in \mathbb{N}\right\}\right)\right] \\
& \quad \times \int_{0}^{T} k(t) d t+M_{2} \sum_{k=1}^{m}\left[\left(d_{k}^{1,0}+d_{k}^{1,2}\right) \beta\left(\left\{x_{n}: n \in \mathbb{N}\right\}\right)\right. \\
& \left.+d_{k}^{1,1} \beta\left(\left\{y_{n}: n \in \mathbb{N}\right\}\right)\right]
\end{aligned}
$$

$$
\begin{aligned}
& +M \sum_{k=1}^{m}\left[\left(d_{k}^{2,0}+d_{k}^{2,2}\right) \beta\left(\left\{x_{n}: n \in \mathbb{N}\right\}\right)\right. \\
& \left.\quad+d_{k}^{2,1} \beta\left(\left\{y_{n}: n \in \mathbb{N}\right\}\right)\right] \\
& \leq\left[M_{2} \ell_{1}+4 M \int_{0}^{T} k(t) d t+M_{2} N_{1}+M N_{2}\right] \\
& \quad \times \beta\left(\left\{x_{n}: n \in \mathbb{N}\right\}\right) \\
& +\left[2 M \int_{0}^{T} k(t) d t+M_{2} N_{3}+M N_{4}\right] \beta\left(\left\{y_{n}: n \in \mathbb{N}\right\}\right) \\
& \leq N_{6} \beta\left(\left\{\left(x_{n}, y_{n}\right): n \in \mathbb{N}\right\}\right) .
\end{aligned}
$$

Finally, collecting these estimates, we get

$$
\begin{aligned}
& \beta\left(\left\{\left(x_{n}, y_{n}\right): n \in \mathbb{N}\right\}\right) \\
& \quad \leq \beta(\Omega) \leq \beta\left(\left\{w_{n}: n \in \mathbb{N}\right\}\right) \\
& \quad \leq \max \left\{\beta\left(\left\{u_{n}: n \in \mathbb{N}\right\}\right), \beta\left(\left\{v_{n}: n \in \mathbb{N}\right\}\right)\right\} \\
& \quad \leq N_{7} \beta\left(\left\{\left(x_{n}, y_{n}\right): n \in \mathbb{N}\right\}\right) .
\end{aligned}
$$

This implies that $\beta(\Omega)=\beta\left(\left\{\left(x_{n}, y_{n}\right): n \in \mathbb{N}\right\}\right)=0$, which in turn implies that $\mathscr{F}$ is a $\beta$-condensing map.

Corollary 19. Under the hypotheses of Theorem 18, there exists a mild solution of problems (1)-(4).

Proof. It follows from Theorem 18 and Theorem 6 that there is a fixed point $(x, y)$ of $\mathscr{F}$. It is follows from (17), (39), and (40) that $x(\cdot) \in \operatorname{PC}^{1}([-h, T] ; X)$ and that $x(\cdot)$ is a fixed point of $\Gamma$.

The sine functions $S(t)$ involved in concrete problems are frequently compact. This allows us to reduce the conditions to obtain the existence of mild solutions to problems (1)-(4). To establish this result some previous properties about sine operators are needed.

Lemma 20. Assume that $S(t)$ is a compact operator for all $t \in$ $\mathbb{R}$. If $D \subset X$ is a bounded set, then the set $\{S(\cdot) x: x \in D\}$ is relatively compact in $C([0, T] ; X)$.

Proof. The set $S(t)(D)$ is relatively compact in $X$ for all $t \epsilon$ $[0, T]$. Moreover, for fixed $t \in[0, T]$ and $s \in \mathbb{R}$ such that $t+s \in[0, T]$ we can decompose

$$
\begin{aligned}
S(t+s) & x-S(t) x \\
= & S(t) C(s) x+S(s) C(t) x-S(t) x \\
= & (C(s)-I) S(t) x+S(s) C(t) x .
\end{aligned}
$$

If we restrict us to consider $x \in D$, using that $S(t)(D)$ is relatively compact, $C(t)(D)$ is bounded, and $\|S(s)\| \leq M s$, we obtain that $(C(s)-I) S(t) x \rightarrow 0$ and $S(s) C(t) x \rightarrow 0$ when $s \rightarrow 0$ uniformly for $x \in D$. Consequently, the set $\{S(\cdot) x$ : $x \in D\}$ is equicontinuous, and the Ascoli-Arzelá theorem 
implies that $\{S(\cdot) x: x \in D\}$ is relatively compact in $C([0, T]$; $X)$.

Lemma 21. Assume that $S(t)$ is a compact operator for all $t \in$ $\mathbb{R}$. Then the map $\Lambda_{1}$ is compact.

Proof. Let $W \subset L^{1}([0, T] ; X)$ be a bounded set. It follows from [40, Theorem 5] that the set $\left\{\Lambda_{1}(u)(t): u \in W\right\}$ is relatively compact in $X$ for every $t \in[0, T]$. On the other hand, using again (56) we can write

$$
\begin{aligned}
&\left\|\Lambda_{1}(u)(t+s)-\Lambda_{1}(u)(t)\right\| \\
&=\left\|\int_{0}^{t+s} S(t+s-\xi) u(\xi) d \xi-\int_{0}^{t} S(t-\xi) u(\xi) d \xi\right\| \\
& \leq\left\|(C(s)-I) \int_{0}^{t} S(t-\xi) u(\xi) d \xi\right\| \\
&+\left\|S(s) \int_{0}^{t} C(t-\xi) u(\xi) d \xi\right\| \\
&+\left\|\int_{t}^{t+s} S(t+s-\xi) u(\xi) d \xi\right\| \\
& \leq\left\|(C(s)-I) \int_{0}^{t} S(t-\xi) u(\xi) d \xi\right\|^{T} \\
&+M^{2}|s| \int_{0}^{T}\|u(\xi)\| d \xi\|+M|s|\| \int_{0}^{T}\|u(\xi)\| d \xi .
\end{aligned}
$$

Since $\left\{\Lambda_{1}(u)(t): u \in W\right\}$ is relatively compact in $X, \|(C(s)-$ I) $\int_{0}^{t} S(t-\xi) u(\xi) d \xi \| \rightarrow 0$ as $s \rightarrow 0$ uniformly for $u \in W$. Combining with the above estimate, it follows that $\Lambda_{1}(u)(t+$ $s)-\Lambda_{1}(u)(t) \rightarrow 0$ as $s \rightarrow 0$ uniformly for $u \in W$. Therefore, the set $\Lambda_{1}(W)$ is equicontinuous. The Ascoli-Arzelá theorem shows that $\Lambda_{1}$ is a compact operator.

We define the constants

$$
\begin{gathered}
N_{5}^{\prime}=\max \left\{\max _{-h \leq \theta \leq 0} \ell(\theta), M\left(\ell(0)+N_{1}+N_{3}\right)\right\}, \\
N_{7}^{\prime}=\max \left\{N_{5}^{\prime}, N_{6}\right\} .
\end{gathered}
$$

Corollary 22. Assume that the operator $S(t)$ is compact for all $t \in \mathbb{R}$. Assume further that $\varphi(0) \in E$ and that conditions (F1)(F4), (g1)-(g3), and (I1)-(I2) hold. If $N_{7}^{\prime}<1$, then there exists a mild solution of problems (1)-(4).

Proof. We repeat the construction carried out in the proof of Theorem 18. The only modification is related with the estimate of $\beta\left(\left\{u_{n}(\cdot): n \in \mathbb{N}\right\}\right)$ for $u_{n}$ defined on $[0, T]$. Using Lemmas 20 and 21 we can see that

$$
\begin{aligned}
& \beta\left(\left\{u_{n}(\cdot): n \in \mathbb{N}\right\}\right) \\
& \leq M \ell(0) \beta\left(\left\{x_{n}: n \in \mathbb{N}\right\}\right) \\
&+M \sum_{k=1}^{m}\left[\left(d_{k}^{1,0}+d_{k}^{1,2}\right) \beta\left(\left\{x_{n}: n \in \mathbb{N}\right\}\right)\right. \\
&\left.+d_{k}^{1,1} \beta\left(\left\{y_{n}: n \in \mathbb{N}\right\}\right)\right]
\end{aligned}
$$

$$
\begin{aligned}
\leq & M\left[\ell(0)+N_{1}\right] \beta\left(\left\{x_{n}: n \in \mathbb{N}\right\}\right) \\
& +M N_{3} \beta\left(\left\{y_{n}: n \in \mathbb{N}\right\}\right) \\
\leq & M\left[\ell(0)+N_{1}+N_{3}\right] \beta\left(\left\{\left(x_{n}, y_{n}\right): n \in \mathbb{N}\right\}\right) .
\end{aligned}
$$

Combining with (45), for $u_{n}$ defined on $[-h, T]$, we obtain

$$
\beta\left(\left\{u_{n}(\cdot): n \in \mathbb{N}\right\}\right) \leq N_{5}^{\prime} \beta\left(\left\{\left(x_{n}, y_{n}\right): n \in \mathbb{N}\right\}\right) .
$$

Proceeding as in the proof of Theorem 18 and Corollary 19, we get that $\Gamma$ has a fixed point $x$, which is a mild solution of problems (1)-(4).

We now are concerned with the following particular case of problems (1)-(4):

$$
\begin{gathered}
x^{\prime \prime}(t)-A x(t) \in F\left(t, x(t), x_{t}\right), \\
t \in J=[0, T], t \neq t_{k}, \quad k=1, \ldots, m, \\
\Delta x\left(t_{k}\right)=I_{k}\left(x\left(t_{k}\right), x_{t_{k}}\right), \quad k=1, \ldots, n, \\
x(\theta)+g(x)(\theta)=\varphi(\theta), \quad \theta \in[-h, 0], \quad x^{\prime}(0)=z .
\end{gathered}
$$

From an intuitive viewpoint this model corresponds to an incomplete second order equation in which the impulses on the path do not lead to changes in the velocity.

We can reduce this problem to a particular case of problems (1)-(4) taking $I_{k}$ as $I_{k}^{1}$ with $I_{k}^{2}=0$ and modifying slightly the conditions about $F, I_{k}$, and $g$. We assume that $F$ is a multivalued map from $J \times X \times C_{h}^{p}$ into $\mathscr{K} v(X)$ that satisfies conditions (F1)-(F4) (now we omit the variable $y$ in these conditions). Proceeding as in Remark 13, for $x(\cdot) \in \mathrm{PC}([-h, T] ; X)$ the function $[0, T] \rightarrow \mathscr{K} v(X), t \mapsto$ $F\left(t, x(t), x_{t}\right)$ admits a Bochner integrable selection. As a consequence, the set

$$
\mathcal{S}_{F, x}=\left\{f \in L^{1}(J ; X): f(t) \in F\left(t, x(t), x_{t}\right), t \in J\right\} \neq \emptyset,
$$

and $\mathcal{S}_{F, x}$ is convex.

Next we describe the conditions on the function $g$. We assume that $g$ is a map from $\mathrm{PC}([-h, T] ; X)$ into $C([-h, 0] ; X)$ that satisfies the following.

(g1) The function $g$ is continuous and takes bounded sets in $\mathrm{PC}([-h, T] ; X)$ into bounded subsets of $C([-h, 0] ; X)$.

(g2) There is a continuous function $\ell:[-h, 0] \rightarrow[0, \infty)$ such that

$$
\chi(g(W)(\theta)) \leq \ell(\theta) \sup _{t \in[-h, T]} \chi(W(t)), \quad \theta \in[-h, 0]
$$

for all bounded sets $W \subset \mathrm{PC}([-h, T], X)$.

(g3) For each bounded set $W \subset \mathrm{PC}([-h, T] ; X)$ the set $g(W)$ is equicontinuous. 
Next we establish the conditions on maps $I_{k}, k=1, \ldots, m$. We assume that $I_{k}: X \times C_{h}^{p} \rightarrow X$ satisfy the following conditions.

(I1) The maps $I_{k}, k=1, \ldots, m$ are continuous and takes bounded sets into bounded sets.

(I2) There are positive constants $d_{k}^{j}, j=1,2, k=1, \ldots, m$, such that

$$
\chi\left(I_{k}\left(D_{1} \times W\right)\right) \leq d_{k}^{1} \chi\left(D_{1}\right)+d_{k}^{2} \sup _{-h \leq \theta \leq 0} \chi(W(\theta))
$$

for all bounded sets $D_{1} \subset X$ and $W \subset C_{h}^{p}$ such that $\sup _{-h \leq \theta \leq 0}\{\|\psi(\theta)\|: \psi \in W\}<\infty$.

We now establish our concept of mild solution.

Definition 23. A function $x(\cdot) \in \mathrm{PC}([-h, T] ; X)$ is said to be a mild solution of (61)-(63) if conditions (62)-(63) are satisfied, and the integral equation

$$
\begin{aligned}
x(t)= & C(t)(\varphi(0)-g(x)(0))+S(t) z \\
& +\int_{0}^{t} S(t-s) f(s) d s \\
& +\sum_{t_{k}<t} C\left(t-t_{k}\right) I_{k}\left(x\left(t_{k}\right), x\left(t_{k}\right)\right)
\end{aligned}
$$

is verified for $f \in \mathcal{S}_{F, x}$ and all $t \in[0, T]$.

We next define the solution map associated with our concept of mild solution for problems (61)-(63) as follows. Let $x \in \mathrm{PC}([-h, T] ; X)$. We define $\Gamma(x)$ to be the set formed by all functions $u$ given by

$$
\begin{aligned}
& u(t) \\
& = \begin{cases}\varphi(t)-g(x)(t), & t \in[-h, 0], \\
C(t)(\varphi(0)-g(x)(0))+S(t) z+\int_{0}^{t} S(t-s) f(s) d s & \\
+\sum_{t_{k}<t} C\left(t-t_{k}\right) I_{k}^{1}\left(x\left(t_{k}\right), x^{\prime}\left(t_{k}\right), x_{t_{k}}\right), & t \in[0, T],\end{cases}
\end{aligned}
$$

for $f \in \mathcal{S}_{F, x}$. It follows from our hypotheses that $u \in \mathrm{PC}([-h, T] ; X)$. Hence, $\Gamma: \operatorname{PC}([-h, T] ; X) \rightarrow$ $\mathscr{P}(\mathrm{PC}([-h, T] ; X))$. Furthermore, it is clear that $x(\cdot)$ is a mild solution of problems (61)-(63) if and only if $x(\cdot)$ is a fixed point of $\Gamma$.

We define

$$
\begin{aligned}
N_{7}^{\prime \prime}=\max \left\{\max _{-h \leq \theta \leq 0} \ell(\theta),\right. & \\
& \left(M\left[\ell(0)+\sum_{k=1}^{m}\left(d_{k}^{1}+d_{k}^{2}\right)\right]\right. \\
& \left.\left.+4 M_{1} \int_{0}^{T} k(t) d t\right)\right\} .
\end{aligned}
$$

We are now in a position to prove the following result.
Theorem 24. Assume that conditions (F1)-(F4), (g1)-(g3), and (I1)-(I2) hold. If $N_{7}^{\prime \prime}<1$, then the map $\Gamma: P C([-h$, $T] ; X) \rightarrow K v(P C([-h, T] ; X))$ is u.s.c. and $\beta$-condensing.

Proof. We proceed as in the proof of Theorem 18. We only include here a sketch of the proof. To prove that $\Gamma$ is $\beta$ condensing. Let $\Omega \subset \mathrm{PC}([-h, T] ; X)$ be a bounded set such that $\beta(\Gamma(\Omega)) \geq \beta(\Omega)$. It follows from Lemma 10 that there exists a sequence $\left(u_{n}\right)_{n}$ in $\Gamma(\Omega)$ such that $\beta(\Gamma(\Omega))=\beta\left(\left\{u_{n}\right.\right.$ : $n \in \mathbb{N}\})$. We can write $u_{n} \in \Gamma\left(x_{n}\right)$ for some $x_{n} \in \Omega$.

To estimate $\beta\left(\left\{u_{n}: n \in \mathbb{N}\right\}\right)$, using (68) we can write

$$
\begin{aligned}
& u_{n}(t) \\
& = \begin{cases}\varphi(t)-g\left(x_{n}\right)(t), & t \in[-h, 0], \\
C(t)\left(\varphi(0)-g\left(x_{n}\right)(0)\right)+S(t) z+\Lambda_{1}\left(f_{n}\right)(t) & \\
+\sum_{t_{k}<t} C\left(t-t_{k}\right) I_{k}\left(x_{n}\left(t_{k}\right),\left(x_{n}\right)_{t_{k}}\right), & t \in[0, T],\end{cases}
\end{aligned}
$$

for $f_{n} \in \mathcal{S}_{F, x_{n}}$.

From (45), we have

$$
\beta\left(\left\{\varphi-g\left(x_{n}\right): n \in \mathbb{N}\right\}\right) \leq \max _{-h \leq \theta \leq 0} \ell(\theta) \beta\left(\left\{x_{n}: n \in \mathbb{N}\right\}\right) .
$$

Now we consider functions $u_{n}$ defined on $[0, T]$. From (70) and using Lemma 7, we get

$$
\begin{aligned}
& \beta\left(\left\{u_{n}(\cdot): n \in \mathbb{N}\right\}\right) \\
& \leq M \chi\left(\left\{g\left(x_{n}\right)(0): n \in \mathbb{N}\right\}\right)+\beta\left(\left\{\Lambda_{1}\left(f_{n}\right)(\cdot): n \in \mathbb{N}\right\}\right) \\
& \quad+M \sum_{k=1}^{m} \chi\left(\left\{I_{k}\left(x_{n}\left(t_{k}\right),\left(x_{n}\right)_{t_{k}}\right): n \in \mathbb{N}\right\}\right) .
\end{aligned}
$$

Using now conditions (g2), (I2), and Remark 14, we have

$$
\begin{aligned}
& \chi\left(\left\{g\left(x_{n}\right)(0): n \in \mathbb{N}\right\}\right) \\
& \quad \leq \ell(0) \beta\left(\left\{x_{n}: n \in \mathbb{N}\right\}\right), \\
& \chi\left(\left\{I_{k}\left(x_{n}\left(t_{k}\right),\left(x_{n}\right)_{t_{k}}\right): n \in \mathbb{N}\right\}\right) \\
& \quad \leq d_{k}^{1} \chi\left(\left\{x_{n}\left(t_{k}\right): n \in \mathbb{N}\right\}\right)+d_{k}^{2} \beta\left(\left\{x_{n}: n \in \mathbb{N}\right\}\right) \\
& \quad \leq\left(d_{k}^{1}+d_{k}^{2}\right) \beta\left(\left\{x_{n}: n \in \mathbb{N}\right\}\right) .
\end{aligned}
$$

On the other hand, since $f_{n} \in \mathcal{S}_{F, x_{n}}$, for $t \in[0, T]$, we have that $f_{n}(t) \in F\left(t, x_{n}(t),\left(x_{n}\right)_{t}\right)$. This implies that $\left\{f_{n}: n \in \mathbb{N}\right\}$ is uniformly integrable and, applying condition (F4),

$$
\begin{aligned}
\chi\left(\left\{f_{n}(t): n \in \mathbb{N}\right\}\right) \\
\leq k(t)\left[\chi\left(\left\{x_{n}(t): n \in \mathbb{N}\right\}\right)\right. \\
\left.\quad+\sup _{-h \leq \theta \leq 0} \chi\left(\left\{x_{n}(t+\theta): n \in \mathbb{N}\right\}\right)\right] \\
\leq 2 k(t) \beta\left(\left\{x_{n}: n \in \mathbb{N}\right\}\right) .
\end{aligned}
$$


Combining this estimate with Lemma 11 we infer that

$$
\beta\left(\left\{\Lambda_{1}\left(f_{n}\right)(\cdot): n \in \mathbb{N}\right\}\right) \leq 4 M_{1} \beta\left(\left\{x_{n}: n \in \mathbb{N}\right\}\right) \int_{0}^{T} k(t) d t
$$

Substituting this estimate in (72), we obtain

$$
\begin{aligned}
\beta( & \left.\left\{u_{n}(\cdot): n \in \mathbb{N}\right\}\right) \\
\leq & \left(M\left[\ell(0)+\sum_{k=1}^{m}\left(d_{k}^{1}+d_{k}^{2}\right)\right]+4 M_{1} \int_{0}^{T} k(t) d t\right) \\
& \times \beta\left(\left\{x_{n}: n \in \mathbb{N}\right\}\right) \\
= & N_{7}^{\prime \prime} \beta\left(\left\{x_{n}: n \in \mathbb{N}\right\}\right) .
\end{aligned}
$$

Collecting these assertions, we get

$$
\beta\left(\left\{x_{n}: n \in \mathbb{N}\right\}\right) \leq \beta(\Gamma(\Omega)) \leq N_{7}^{\prime \prime} \beta\left(\left\{x_{n}: n \in \mathbb{N}\right\}\right),
$$

which implies that $\beta(\Gamma(\Omega))=0$, which in turn shows that $\Gamma$ is $\beta$-condensing and completes the proof.

The following assertions are immediate consequences of Theorem 24.

Corollary 25. Under the hypotheses of Theorem 24, there exists a mild solution of problems (61)-(63).

Corollary 26. Assume that the operator $S(t)$ is compact for all $t \in \mathbb{R}$. Assume further that conditions (F1)-(F4), (g1)-(g3), and (I1)-(I2) hold. If

$$
\max \left\{\max _{-h \leq \theta \leq 0} \ell(\theta), M\left[\ell(0)+\sum_{k=1}^{m}\left(d_{k}^{1}+d_{k}^{2}\right)\right]\right\}<1,
$$

then there exists a mild solution of problems (61)-(63).

\section{Applications}

In this section we apply our abstract results to study the existence of solutions to the impulsive retarded wave equation described by (5)-(9). To model this problem in abstract form, in what follows we consider the space $X=L^{2}([0, \pi])$ and $A$ : $D(A) \subseteq X \rightarrow X$ is the map defined by $A x=\left(d^{2} / d \xi^{2}\right) x(\xi)$ with domain $D(A)=\left\{x \in X: x^{\prime \prime} \in X, x(0)=x(\pi)=0\right\}$. It is well known that $A$ is the infinitesimal generator of a strongly continuous cosine function $(C(t))_{t \in \mathbb{R}}$ on $X$. Furthermore, $A$ has a discrete spectrum and the eigenvalues are $-n^{2}, n \in \mathbb{N}$, with corresponding eigenvectors $z_{n}(\xi)=(2 / \pi)^{1 / 2} \sin (n \xi)$. Furthermore, the set $\left\{z_{n}: n \in \mathbb{N}\right\}$ is an orthonormal basis of $X$ and the following properties hold.

(a) For $x \in D(A), A x=-\sum_{n=1}^{\infty} n^{2}\left\langle x, z_{n}\right\rangle z_{n}$.

(b) For $x \in X$,

$$
\begin{aligned}
& C(t) x=\sum_{n=1}^{\infty} \cos (n t)\left\langle x, z_{n}\right\rangle z_{n} \\
& S(t) x=\sum_{n=1}^{\infty} \frac{\sin (n t)}{n}\left\langle x, z_{n}\right\rangle z_{n} .
\end{aligned}
$$

Consequently, $\|C(t)\|=\|S(t)\| \leq 1$ for all $t \in \mathbb{R}$ and $S(t)$ is a compact operator for every $t \in \mathbb{R}$.

(c) The space $E=\left\{x \in H^{1}(0, \pi): x(0)=x(\pi)=0\right\}$ (see [30] for details) and $\|x\|_{E} \leq\|x\|+\left\|x^{\prime}\right\|$. In particular, we observe that the inclusion $\iota: E \rightarrow X$ is compact. Moreover, the function $S(\cdot)$ is $2 \pi$-periodic. Using this property and (13) we can show that

$$
\|A S(t)\|_{\mathscr{L}(E ; X)} \leq 2, \quad \forall t \in \mathbb{R} .
$$

In fact, using the periodicity of $S(\cdot)$ is sufficient to establish the property for $t \in[-\pi, \pi]$. It is an immediate consequence of the definition of the norm in $E$ that $\|A S(t)\|_{\mathscr{L}(E ; X)} \leq 1$, for $t \in[0,1]$. For $t \in[1, \pi / 2]$, we can write $t=1+s$ with $s \in[0,1]$, and using (13) we have

$$
A S(t)=A S(1+s)=C(1) A S(s)+C(s) A S(1) .
$$

Since $\|C(\tau)\| \leq 1$ for all $\tau \in \mathbb{R}$, we obtain

$$
\begin{aligned}
\|A S(t)\|_{\mathscr{L}(E ; X)} & \\
\leq & \|C(1)\|\|A S(s)\|_{\mathscr{L}(E ; X)} \\
& \quad+\|C(s)\|\|A S(1)\|_{\mathscr{L}(E ; X)} \leq 2 .
\end{aligned}
$$

Similarly, for $t \in[\pi / 2, \pi]$, we can write $t=\pi-s$ with $s \in$ $[0, \pi / 2]$, and using again (13), we can write

$$
\begin{aligned}
\operatorname{AS}(t) & =A S(\pi-s) \\
& =C(s) A S(\pi)-C(\pi) A S(s)=-C(\pi) A S(s),
\end{aligned}
$$

which implies

$$
\|A S(t)\|_{\mathscr{L}(E ; X)} \leq\|C(\pi)\|\|A S(s)\|_{\mathscr{L}(E ; X)} \leq 2 .
$$

In view of that $A S(-t)=-A S(t)$, this completes the proof of the assertion.

In what follows we assume that $z \in X$ and that $\varphi \in$ $C([-h, 0] ; X)$, where we have identified $\varphi(\theta)(\xi)=\varphi(\theta, \xi)$ for $\theta \in[-h, 0]$ and $\xi \in[0, \pi]$.

Initially we construct the multivalued function $F$. We assume that $f_{0}: J \times[0, \pi] \times \mathbb{R}^{3} \rightarrow \mathscr{K} v(\mathbb{R})$ is a bounded multivalued map that satisfies the following conditions.

(f1) There exist positive constants $L, L_{1}$, and $L_{2}$ such that

$$
\begin{aligned}
& d_{H}\left(f_{0}\left(t_{1}, \xi_{1}, \phi_{1}\right), f_{0}\left(t_{2}, \xi_{2}, \phi_{2}\right)\right)^{2} \\
& \quad \leq L^{2}\left|t_{1}-t_{2}\right|^{2}+L_{1}^{2}\left|\xi_{1}-\xi_{2}\right|^{2}+L_{2}^{2}\left\|\phi_{1}-\phi_{2}\right\|^{2},
\end{aligned}
$$

for all $\left(t, \xi_{1}, \phi_{1}\right),\left(t, \xi_{2}, \phi_{2}\right) \in J \times[0, \pi] \times \mathbb{R}^{3}$, where $d_{H}$ denotes the Hausdorff metric and $\|\cdot\|$ denotes the Euclidean norm in $\mathbb{R}^{3}$.

(f2) There exists a positive function $\mu \in L^{1}([0, T])$ such that $|s| \leq \mu(t)$ for all $s \in f_{0}(t, 0)$.

In a metric space $(\Omega, d)$, we denote $\rho(w, B)=\inf \{d(w, b)$ : $b \in B\}$. We will use the following property of the Hausdorff metric. 
Lemma 27. Let $(\Omega, d)$ be a metric space, and let $B_{1}, B_{2} \subseteq \Omega$ be bounded sets. Then, for every $u, v \in \Omega$,

$$
\rho\left(v, B_{2}\right) \leq d(v, u)+\rho\left(u, B_{1}\right)+d_{H}\left(B_{1}, B_{2}\right) .
$$

We have the following consequences.

Proposition 28. Under the previous conditions, the following properties hold.

(i) For each $t \in J$ and $\phi \in \mathbb{R}^{3}$, the function $f_{0}(t, \cdot, \phi)$ is measurable.

(ii) For each $t \in J$ and $\xi \in[0, \pi]$, the function $f_{0}(t, \xi, \cdot)$ is upper semicontinuous.

(iii) For each $t \in J$ and $\phi \in L^{2}\left([0, \pi] ; \mathbb{R}^{3}\right)$ the set

$$
\mathscr{T}_{f_{0}, \phi}(t)=\left\{w \in L^{2}([0, \pi]): w(\xi) \in f_{0}(t, \xi, \phi)\right\} \neq \emptyset
$$

is closed convex in $L^{2}([0, \pi])$.

Proof. Consider the following.

(i) It is an immediate consequence of the fact that the multivalued map $f_{0}(t, \cdot, \phi)$ is $d_{H^{-}}$continuous.

(ii) We know that $f_{0}\left(t, \xi, \mathbb{R}^{3}\right)$ is bounded and, therefore, relatively compact. We will show that the graph $f(t, \xi, \cdot)$ is closed. Assume that $\phi_{n}, \phi \in \mathbb{R}^{3}$ and $\phi_{n} \rightarrow \phi, s_{n} \in f_{0}\left(t, \xi, \phi_{n}\right), s_{n} \rightarrow s$ as $n \rightarrow \infty$. Using Lemma 27 we can write

$$
\begin{aligned}
& \rho\left(s, f_{0}(t, \xi, \phi)\right) \\
& \quad \leq\left|s-s_{n}\right|+d_{H}\left(f_{0}\left(t, \xi, \phi_{n}\right), f_{0}(t, \xi, \phi)\right) .
\end{aligned}
$$

Since $f_{0}(t, \xi, \cdot)$ is $d_{H}$-continuous, it follows that $\rho\left(s, f_{0}(t, \xi, \phi)\right)=0$. In view of that the set $f_{0}(t, \xi, \phi)$ is closed, we conclude that $s \in f_{0}(t, \xi, \phi)$. Applying [3, Proposition 1.2] we obtain that $f_{0}(t, \xi, \cdot)$ is upper semicontinuous.

(iii) For $t \in J$ and $\phi \in L^{2}\left([0, \pi] ; \mathbb{R}^{3}\right)$ the map $f_{0}(t, \cdot, \phi)$ : $[0, \pi] \rightarrow \mathscr{K} v(\mathbb{R}), \xi \mapsto f_{0}(t, \xi, \phi(\xi))$, is measurable with closed values. It follows from [3, Proposition 3.2] that there exists a measurable selection $w$ such that $w(\xi) \in f_{0}(t, \xi, \phi(\xi))$. Using that $f$ is bounded it follows that $w \in L^{2}([0, \pi])$. This shows that $\mathscr{T}_{f_{0}, \phi}(t) \neq \emptyset$. Since the values of $f_{0}$ are convex, it follows that $\mathscr{T}_{f_{0}, \phi}(t)$ is also convex.

To establish that $\mathscr{T}_{f_{0}, \phi}(t)$ is closed, we consider a sequence $w_{n}, w \in L^{2}([0, \pi]), w_{n} \in \mathscr{T}_{f_{0}, \phi}(t)$ such that $w_{n} \rightarrow$ $w, n \rightarrow \infty$, for the norm in $L^{2}([0, \pi])$. By passing to a subsequence if necessary, we can assume that $w_{n}(\xi) \rightarrow w(\xi), n \rightarrow$ $\infty$, a.e. $\xi \in[0, \pi]$. Since $f_{0}(t, \xi, \phi(\xi))$ is closed, it follows that $w(\xi) \in f_{0}(t, \xi, \phi(\xi))$, which in turn implies that $w \in$ $\mathscr{T}_{f_{0}, \phi}(t)$.

For $\phi \in L^{2}\left([0, \pi] ; \mathbb{R}^{3}\right)$ we define

$$
\mathscr{T}_{f_{0}, \phi}^{1}(t)=\left\{\int_{0}^{\xi} w(\eta) d \eta: w \in \mathscr{T}_{f_{0}, \phi}(t)\right\},
$$

and $F_{1}(\phi): J \rightarrow \mathscr{P}(X)$ is given by $F_{1}(\phi)(t)=\mathscr{T}_{f_{0}, \phi}^{1}(t)$.
Proposition 29. Under the previous conditions, $F_{1}$ is a measurable and upper semicontinuous map with convex compact values.

Proof. Initially we show that $\mathscr{T}_{f_{0}, \phi}^{1}(t)$ is closed. Let $v_{n}(\xi)=$ $\int_{0}^{\xi} w_{n}(\eta) d \eta$ be a sequence in $\mathscr{T}_{f_{0}, \phi}^{1}(t)$ that converges to $v$ as $n \rightarrow \infty$. Since $\mathscr{T}_{f_{0}, \phi}(t)$ is sequentially weakly compact, there is $w \in L^{2}([0, \pi])$ and a subsequence $w_{n_{k}}$ such that $w_{n_{k}} \rightarrow w$ as $k \rightarrow \infty$ in the weak topology. Since $\mathscr{T}_{f_{0}, \phi}(t)$ is a closed convex set, it follows that $w \in \mathscr{T}_{f_{0}, \phi}(t)$. In view of that

$$
\begin{aligned}
& \int_{0}^{\xi} w_{n_{k}}(\eta) d \eta-\int_{0}^{\xi} w(\eta) d \eta \\
& \quad=\int_{0}^{\pi}\left(w_{n_{k}}-w\right) \chi_{[0, \xi]}(\eta) d \eta \longrightarrow 0, \quad k \longrightarrow \infty
\end{aligned}
$$

where $\chi_{[0, \xi]}$ denotes the characteristic function of the interval $[0, \xi]$, we obtain that $v(\xi)=\int_{0}^{\xi} w(\eta) d \eta$ and $v \in \mathscr{T}_{f_{0}, \phi}^{1}(t)$.

Since the functions in $\mathscr{T}_{f_{0}, \phi}(t)$ are uniformly bounded,

$$
\begin{aligned}
& \int_{0}^{\pi}|v(\xi+\delta)-v(\xi)|^{2} d \xi \\
& \quad=\int_{0}^{\pi}\left|\int_{\xi}^{\xi+\delta} w(\eta) d \eta\right|^{2} d \xi \leq \pi \sup _{0 \leq \xi \leq \pi}|w(\xi)|^{2} \delta^{2}
\end{aligned}
$$

converge to zero as $\delta \rightarrow 0$ uniformly for $v \in \mathscr{T}_{f_{0}, \phi}^{1}(t)$. From [41, Theorem IV.8.20] we conclude that $\mathscr{T}_{f_{0}, \phi}^{1}(t)$ is relatively compact.

On the other hand, proceeding as in the proof of Proposition 28(ii), we get that $F_{1}(\phi)$ is upper semicontinuous. Finally, as a consequence of a remark in [5, page 21], we can affirm that $F_{1}(\phi)$ is measurable. tion.

The following consequence is essential for our construc-

Corollary 30. Under the above conditions, there exists a measurable selection for $F_{1}(\phi)$.

Proof. It follows from [3, Proposition 3.2].

We now consider the map $F: J \times X \times X \times C_{h}^{2} \rightarrow \mathscr{K} v(X)$ defined by

$$
F(t, x, y, \psi)=\mathscr{T}_{f_{0}, \phi}^{1}(t)
$$

for $\phi=\left(x, y, \int_{-h}^{0} \psi(\theta) d \theta\right)$. Since $F(\cdot, x, y, \psi)=F_{1}(\phi)(\cdot)$, it follows from our construction that $F$ satisfies condition $(\mathrm{F} 1)$. Moreover, proceeding as in the proof of Proposition 28(ii) we conclude that $F$ is upper semicontinuous, which shows that $F$ satisfies condition (F2). On the other hand, if $v \in F(t, x, y, \psi)$, then there exists $w \in \mathscr{T}_{f_{0}, \phi}(t)$ such that $v(\xi)=\int_{0}^{\xi} w(\eta) d \eta$ 
with $w(\xi) \in f_{0}\left(t, \xi, x(\xi), y(\xi), \int_{-h}^{0} \psi(\theta, \xi) d \theta\right)$. Therefore, there exists $w^{1}(\xi) \in f_{0}(t, 0)$ such that

$$
\begin{aligned}
& |w(\xi)| \\
& \leq \mu(t)+1 \\
& \quad+d_{H}\left(f_{0}(t, \xi, x(\xi), y(\xi),\right. \\
& \left.\left.\quad\left(\int_{-h}^{0} \psi(\theta, \xi) d \theta\right)\right), f_{0}(t, 0)\right) \\
& \leq \mu(t)+1+\left(L_{1}^{2} \xi^{2}+L_{2}^{2}\left(x(\xi)^{2}+y(\xi)^{2}\right.\right. \\
& \left.\left.\quad+\left(\int_{-h}^{0} \psi(\theta, \xi) d \theta\right)^{2}\right)\right)^{1 / 2} .
\end{aligned}
$$

Hence

$$
\begin{aligned}
& \int_{0}^{\pi}|v(\xi)|^{2} d \xi \\
& \leq \pi^{2} \int_{0}^{\pi}|w(\xi)|^{2} d \xi \\
& \leq 2 \pi^{2} \int_{0}^{\pi}\left[(\mu(t)+1)^{2}+L_{1}^{2} \xi^{2}+L_{2}^{2}\right. \\
& \left.\times\left(x(\xi)^{2}+y(\xi)^{2}+\left(\int_{-h}^{0} \psi(\theta, \xi) d \theta\right)^{2}\right)\right] d \xi \\
& \leq 2 \pi^{2}\left[\pi(\mu(t)+1)^{2}+L_{1}^{2} \frac{\pi^{3}}{3}+L_{2}^{2}\right. \\
& \left.\times\left(\|x\|^{2}+\|y\|^{2}+\int_{0}^{\pi}\left(\int_{-h}^{0} \psi(\theta, \xi) d \theta\right)^{2}\right) d \xi\right] \\
& \leq 2 \pi^{2}\left[\pi(\mu(t)+1)^{2}+L_{1}^{2} \frac{\pi^{3}}{3}\right. \\
& \left.+L_{2}^{2}\left(\|x\|^{2}+\|y\|^{2}+h^{2}\|\psi\|_{C_{h}^{2}}^{2}\right)\right],
\end{aligned}
$$

which shows that $F$ satisfies the condition (F3).

Proposition 31. Let $x, x^{1}, y, y^{1} \in X, \psi, \psi^{1} \in C_{h}^{2}$, and $v \in$ $F(t, x, y, \psi)$. Then

$$
\begin{aligned}
& \rho\left(v, F\left(t, x^{1}, y^{1}, \psi^{1}\right)\right) \\
& \quad \leq \pi L_{2}\left(\left\|x-x^{1}\right\|^{2}+\left\|y-y^{1}\right\|^{2}+h^{2}\left\|\psi-\psi^{1}\right\|_{C_{h}^{2}}^{2}\right)^{1 / 2} .
\end{aligned}
$$

Proof. To abbreviate the text we introduce the notations $\phi(\xi)=\left(x(\xi), y(\xi), \int_{-h}^{0} \psi(\theta, \xi) d \theta\right)$ and $\phi^{1}(\xi)=\left(x^{1}(\xi), y^{1}(\xi)\right.$, $\left.\int_{-h}^{0} \psi^{1}(\theta, \xi) d \theta\right)$. Since $v \in \mathscr{T}_{f_{0}, \phi}^{1}(t)$, there is $w \in \mathscr{T}_{f_{0}, \phi}(t)$ such that $v(\xi)=\int_{0}^{\xi} w(\eta) d \eta$. Moreover, in view of that $\mathscr{T}_{f_{0}, \phi^{1}}^{1}(t)$ is convex compact in $L^{2}([0, \pi])$, there is $v_{0} \in \mathscr{T}_{f_{0}, \phi^{1}}^{1}(t)$ the nearest point of $v$ in $\mathscr{T}_{f_{0}, \phi^{1}}^{1}(t)$. Consequently, there is $w_{0} \epsilon$ $\mathscr{T}_{f_{0}, \phi^{1}}(t)$ such that $v_{0}(\xi)=\int_{0}^{\xi} w_{0}(\eta) d \eta$. Therefore,

$$
\begin{aligned}
& \rho\left(v, F\left(t, x^{1}, y^{1}, \psi^{1}\right)\right)^{2} \\
& \quad=\left\|v-v_{0}\right\|^{2} \\
& \quad=\int_{0}^{\pi}\left(\int_{0}^{\xi}\left(w(\eta)-w_{0}(\eta)\right) d \eta\right)^{2} d \xi \\
& \leq \pi^{2} \int_{0}^{\pi}\left|w(\eta)-w_{0}(\eta)\right|^{2} d \eta \\
& \leq \pi^{2} L_{2}^{2} \int_{0}^{\pi}\left[\left|x(\eta)-x^{1}(\eta)\right|^{2}+\left|y(\eta)-y^{1}(\eta)\right|^{2}\right. \\
& \left.\quad+\left|\int_{-h}^{0}\left(\psi(\theta, \xi)-\psi^{1}(\theta, \xi)\right) d \theta\right|^{2}\right] d \eta \\
& \leq \pi^{2} L_{2}^{2}\left[\left\|x-x^{1}\right\|^{2}+\left\|y-y^{1}\right\|^{2}+h^{2}\left\|\psi-\psi^{1}\right\|_{C_{h}^{2}}^{2}\right],
\end{aligned}
$$

which completes the proof.

Corollary 32. Under the above conditions, let $\Omega_{i} \subset L^{2}([0, \pi])$, $i=1,2$, be bounded sets, and let $Q \subset C_{h}^{2}$ be a set uniformly bounded. Then

$$
\begin{aligned}
& \chi\left(F\left(t, \Omega_{1}, \Omega_{2}, Q\right)\right) \\
& \quad \leq \pi L_{2}\left(\chi\left(\Omega_{1}\right)^{2}+\chi\left(\Omega_{2}\right)^{2}+h^{2} \gamma(Q)^{2}\right)^{1 / 2},
\end{aligned}
$$

where $\gamma(Q)$ denotes the Hausdorff measure of noncompactness for the norm of uniform convergence.

Proof. Let $\varepsilon>0$. We abbreviate the notation by writing $s_{i}=\chi\left(\Omega_{i}\right)$ and $s=\gamma(Q)$. There exist $x^{1}, \ldots, x^{i_{1}} \in L^{2}([0, \pi])$, $y^{1}, \ldots, y^{j_{1}} \in L^{2}([0, \pi])$, and $\psi^{1}, \ldots, \psi^{k_{1}} \in C_{h}^{2}$ having the following property: given $x \in \Omega_{1}, y \in \Omega_{2}$, and $\psi \in Q$ there are $x^{i}, y^{j}$, and $\psi^{k}$ such that $\left\|x-x^{i}\right\| \leq s_{1}+\varepsilon,\left\|y-y^{j}\right\| \leq s_{2}+\varepsilon$ and $\left\|\psi-\psi^{k}\right\|_{\infty} \leq s+\varepsilon$. Hence, if $v \in F(t, x, y, \psi)$, using Proposition 31, we obtain that

$$
\begin{aligned}
& \rho\left(v, F\left(t, x^{i}, y^{j}, \psi^{k}\right)\right) \\
& \quad \leq \pi L_{2}\left(\left(s_{1}+\varepsilon\right)^{2}+\left(s_{2}+\varepsilon\right)^{2}+h^{2}(s+\varepsilon)^{2}\right)^{1 / 2} .
\end{aligned}
$$

Since $F\left(t, x^{i}, y^{j}, \psi^{k}\right)$ is a compact set in $L^{2}([0, \pi])$, and $\varepsilon>$ 0 was chosen arbitrarily, this completes the proof of the assertion.

Corollary 32 shows that $F$ satisfies the condition (F4). 
On the other hand, we define $g: \operatorname{PC}([-h, T] ; X) \rightarrow$ $C([-h, 0] ; X)$ by

$$
\begin{array}{r}
g(x)(\theta, \xi)=\sigma(\xi) \int_{0}^{T} \int_{0}^{\xi} x(t+\theta, \eta) d \eta d t, \\
x \in \operatorname{PC}([-h, T] ; X),
\end{array}
$$

for $\theta \in[-h, 0]$ and $\xi \in[0, \pi]$. We assume that $\sigma(\cdot)$ is a function of class $C^{1}$ such that $\sigma(\pi)=0$. It is clear that

$$
g(x)(0, \xi)=\sigma(\xi) \int_{0}^{T} \int_{0}^{\xi} x(t, \eta) d \eta d t \in H^{1}(0, \pi)
$$

and $g(x)(0,0)=g(x)(0, \pi)=0$. This implies that $g(x)(0) \in E$ for all $x \in \mathrm{PC}([-h, T] ; X)$. Moreover, $g$ is a continuous map that takes bounded sets into bounded sets, and $g(x)(0)$ : $\mathrm{PC}([-h, T] ; X) \rightarrow E$ is also continuous and takes bounded sets into bounded sets in $E$. This shows that $g$ satisfies condition (g1).

It is clear that $g(x)(\theta)$ is a continuous function from $[0, \pi]$ into $\mathbb{R}$ for each $x \in \mathrm{PC}([-h, T], X)$. Let $W \subset \mathrm{PC}([-h, T], X)$ be a bounded set. It is not difficult to see that the set $\{g(x)(\theta)$ : $x \in W\}$ is equicontinuous. Moreover,

$$
\begin{aligned}
|g(x)(\theta, \xi)| & \leq\|\sigma\|_{\infty}\left|\int_{0}^{T} \int_{0}^{\xi} x(t, \eta) d \eta d t\right| \\
& \leq\|\sigma\|_{\infty} \int_{0}^{T} \int_{0}^{\pi}|x(t, \eta)| d \eta d t \\
& \leq\|\sigma\|_{\infty} \int_{0}^{T} \pi^{1 / 2}\left(\int_{0}^{\pi}|x(t, \eta)|^{2} d \eta\right)^{1 / 2} d t \\
& \leq\|\sigma\|_{\infty} \pi^{1 / 2} T \sup _{-h \leq t \leq T}\|x(t)\|,
\end{aligned}
$$

which shows that $\{g(x)(\theta, \xi): x \in W\}$ is bounded. The Ascoli-Arzelá theorem implies that $\{g(x)(\theta): x \in W\}$ is relatively compact in $C([0, \pi])$. Therefore, $\{g(x)(\theta): x \in W\}$ is also relatively compact in $L^{2}([0, \pi])$. Hence $\chi(g(W)(\theta))=$ 0 , and we can take $\ell(\theta)=0$. This shows that $g$ satisfies the first part of condition (g2). Furthermore, it follows from (c) that

$\chi_{E}(g(W)(0))$

$$
\leq \chi(g(W)(0))+\chi\left(\frac{d}{d \xi} g(W)(0)\right)=\chi\left(\frac{d}{d \xi} g(W)(0)\right) .
$$

From the definition of $g$ we obtain

$$
\begin{aligned}
& \frac{d}{d \xi} g(x)(0, \xi) \\
& \quad=\frac{d}{d \xi} \sigma(\xi) \int_{0}^{T} \int_{0}^{\xi} x(t, \eta) d \eta d t+\sigma(\xi) \int_{0}^{T} x(t, \xi) d t .
\end{aligned}
$$

Arguing as above, we can affirm that the first term on the right hand side of (103) defines a relatively compact set in $X$. Since $\sigma(\xi) \int_{0}^{T} x(t, \xi) d t=\sigma \int_{0}^{T} x(t) d t$ in the space $L^{2}([0, \pi])$, using [42, Theorem 3.1], we conclude that

$$
\begin{aligned}
& \chi\left(\frac{d}{d \xi} g(W)(0)\right) \\
& \quad \leq\|\sigma\|_{\infty} \int_{0}^{T} \chi(W(t)) d t \leq\|\sigma\|_{\infty} T \sup _{t \in[-h, T]} \chi(W(t)),
\end{aligned}
$$

which shows that $g$ also satisfies the second part of condition (g2) with $\ell_{1}=\|\sigma\|_{\infty} T$.

On the other hand,

$$
\begin{aligned}
& \|g(x)(\theta+\delta)-g(x)(\theta)\|^{2} \\
& =\int_{0}^{\pi}|g(x)(\theta+\delta, \xi)-g(x)(\theta, \xi)|^{2} d \xi \\
& =\int_{0}^{\pi} \sigma(\xi)^{2} \mid \int_{0}^{\xi} \int_{0}^{T}(x(t+\theta+\delta, \eta) \\
& -x(t+\theta, \eta))\left.d t d \eta\right|^{2} d \xi \\
& \leq\|\sigma\|_{\infty}^{2} \int_{0}^{\pi} \mid \int_{0}^{\xi}\left[\int_{\theta+\delta}^{T+\theta+\delta} x(s, \eta) d s\right. \\
& \left.-\int_{\theta}^{T+\theta} x(s, \eta) d s\right]\left.d \eta\right|^{2} d \xi \\
& =\|\sigma\|_{\infty}^{2} \int_{0}^{\pi} \mid \int_{0}^{\xi}\left[\int_{T+\theta}^{T+\theta+\delta} x(s, \eta) d s\right. \\
& \left.-\int_{\theta}^{\theta+\delta} x(s, \eta) d s\right]\left.d \eta\right|^{2} d \xi \\
& \leq\|\sigma\|_{\infty}^{2} \int_{0}^{\pi}\left(\int _ { 0 } ^ { \xi } \left[\int_{T+\theta}^{T+\theta+\delta}|x(s, \eta)| d s\right.\right. \\
& \left.\left.+\int_{\theta}^{\theta+\delta}|x(s, \eta)| d s\right] d \eta\right)^{2} d \xi \\
& \leq\|\sigma\|_{\infty}^{2} \int_{0}^{\pi}\left(\int_{T+\theta}^{T+\theta+\delta} \int_{0}^{\pi}|x(s, \eta)| d \eta d s\right. \\
& \left.+\int_{\theta}^{\theta+\delta} \int_{0}^{\pi}|x(s, \eta)| d \eta d s\right)^{2} d \xi \\
& \leq \pi\|\sigma\|_{\infty}^{2} \int_{0}^{\pi}\left(\int_{T+\theta}^{T+\theta+\delta}\|x(s)\| d s+\int_{\theta}^{\theta+\delta}\|x(s)\| d s\right)^{2} d \xi \\
& \leq 4 \pi^{2}\|\sigma\|_{\infty}^{2} \sup _{-h \leq s \leq T}\|x(s)\|^{2} \delta^{2}
\end{aligned}
$$

converges to zero as $\delta \rightarrow 0$ uniformly for $x \in W$. This shows that the set $g(W)$ is equicontinuous. Consequently, we can affirm that $g$ satisfies condition (g3). 


$$
\begin{aligned}
& \text { We define } I_{k}^{1}, I_{k}^{2}: X \times X \times C_{h}^{2} \rightarrow X \text { by } \\
& I_{k}^{1}(x, y, \psi)(\xi)=a_{k}^{1}(\xi) \int_{0}^{\pi} q_{k}^{1}(\eta) x(\eta) d \eta+b_{k}^{1}(\xi), \\
& I_{k}^{2}(x, y, \psi)(\xi)=a_{k}^{2}(\xi) \int_{0}^{\pi} q_{k}^{2}(\eta) y(\eta) d \eta+b_{k}^{2}(\xi),
\end{aligned}
$$

for $x, y \in L^{2}([0, \pi])$, and $\xi \in[0, \pi]$. We assume that $q_{k}^{i}(\cdot), a_{k}^{i}(\cdot), b_{k}^{i}(\cdot) \in L^{2}([0, \pi])$ for $i=1,2$, and that $a_{k}^{1}(\cdot), b_{k}^{1}(\cdot) \in$ $C^{1}([0, \pi])$ are functions such that $a_{k}^{1}(0)=a_{k}^{1}(\pi)=b_{k}^{1}(0)=$ $b_{k}^{1}(\pi)=0$. Proceeding as above, it is easy to see that $I_{k}^{1}$ : $X \times X \times C_{h}^{2} \rightarrow E$ and $I_{k}^{2}: X \times X \times C_{h}^{2} \rightarrow X$ are continuous maps that take bounded sets into bounded sets. This shows that condition (I1) is verified. In addition, using that the map $X \rightarrow \mathbb{R}, y \mapsto \int_{0}^{\pi} q_{k}^{2}(\eta) y(\eta) d \eta$, is a bounded linear functional with norm $\left\|q_{k}^{2}\right\|$, we deduce that

$$
\chi\left(I_{k}^{2}\left(D_{0} \times D_{1} \times W\right)\right) \leq\left\|a_{k}^{2}\right\|\left\|q_{k}^{2}\right\| \chi\left(D_{1}\right) .
$$

Using this argument together with condition (c), we get

$$
\chi\left(I_{k}^{1}\left(D_{0} \times D_{1} \times W\right)\right) \leq\left(\left\|a_{k}^{1}\right\|+\left\|\frac{d}{d \xi} a_{k}^{1}\right\|\right)\left\|q_{k}^{1}\right\| \chi\left(D_{0}\right),
$$

which shows that condition (I2) is also verified.

We complete our model by defining $x(t)=u(t, \cdot)$. It is not difficult to see that under the conditions specified previously, systems (5)-(9) are described by the abstract models (1)-(4). The constants $N_{i}$ introduced in Section 3 are the following:

$$
\begin{aligned}
N_{1}= & \sum_{k=1}^{m}\left(d_{k}^{1,0}+d_{k}^{1,2}\right) \\
= & \sum_{k=1}^{m} d_{k}^{1,0}=\sum_{k=1}^{m}\left(\left\|a_{k}^{1}\right\|+\left\|\frac{d}{d \xi} a_{k}^{1}\right\|\right)\left\|q_{k}^{1}\right\|, \\
N_{2}= & \sum_{k=1}^{m}\left(d_{k}^{2,0}+d_{k}^{2,2}\right)=0, \\
N_{3}= & \sum_{k=1}^{m} d_{k}^{1,1}=0, \\
N_{4}= & \sum_{k=1}^{m} d_{k}^{2,1}=\sum_{k=1}^{m}\left\|a_{k}^{2}\right\|\left\|q_{k}^{2}\right\|, \\
N_{5}^{\prime}= & M\left(\ell(0)+N_{1}+N_{3}\right)=N_{1}, \\
N_{6}= & M \ell_{1}+6 M \int_{0}^{T} k(t) d t+M_{2}\left(N_{1}+N_{3}\right) \\
& +M\left(N_{2}+N_{4}\right) \\
= & \left(2\|\sigma\|_{\infty}+6 \pi L_{2}\right) T+2 N_{1}+N_{4}, \\
N_{7}^{\prime}= & \max \left\{N_{5}^{\prime}, N_{6}\right\}=N_{6} .
\end{aligned}
$$

Combining with Corollary 22, we have established the following result.
Theorem 33. Assume that $\varphi(0, \cdot) \in E$, and

$$
\begin{aligned}
& \left(2\|\sigma\|_{\infty}+6 \pi L_{2}\right) T \\
& \quad+\sum_{k=1}^{m}\left[2\left(\left\|a_{k}^{1}\right\|+\left\|\frac{d}{d \xi} a_{k}^{1}\right\|\right)\left\|q_{k}^{1}\right\|+\left\|a_{k}^{2}\right\|\left\|q_{k}^{2}\right\|\right]<1 .
\end{aligned}
$$

Then there exists a mild solution of systems (5)-(9).

\section{Conflict of Interests}

The authors declare that there is no conflict of interests regarding the publication of this paper.

\section{Acknowledgment}

Hernán R. Henríquez was partially supported by CONICYT under Grants FONDECYT 1130144 and DICYT-USACH.

\section{References}

[1] S. Abbas, M. Benchohra, and G. M. N’Guérékata, Topics in Fractional Differential Equations, Springer, New York, NY, USA, 2012.

[2] M. Benchohra, J. Henderson, and S. Ntouyas, Impulsive Differential Equations and Inclusions, Hindawi Publishing Corporation, New York, NY, USA, 2006.

[3] K. Deimling, Multivalued Differential Equations, vol. 1 of De Gruyter Series in Nonlinear Analysis and Applications, Walter de Gruyter \& Co., Berlin, Germany, 1992.

[4] L. Górniewicz, Topological Fixed Point Theory of Multivalued Mappings, Springer, Dordrecht, The Netherlands, 2nd edition, 2006.

[5] M. Kamenskii, V. Obukhovskii, and P. Zecca, Condensing Multivalued Maps and Semilinear Differential Inclusions in Banach Spaces, vol. 7 of De Gruyter Series in Nonlinear Analysis and Applications, Walter de Gruyter \& Co., Berlin, Germany, 2001.

[6] A. M. Samoĭlenko and N. A. Perestyuk, Impulsive Differential Equations, vol. 14, World Scientific, Singapore, 1995.

[7] N. Abada, M. Benchohra, and H. Hammouche, "Existence and controllability results for nondensely defined impulsive semilinear functional differential inclusions," Journal of Differential Equations, vol. 246, no. 10, pp. 3834-3863, 2009.

[8] M. Benchohra, E. P. Gatsori, L. Górniewicz, and S. K. Ntouyas, "Nondensely defined evolution impulsive differential equations with nonlocal conditions," Fixed Point Theory, vol. 4, no. 2, pp. 185-204, 2003.

[9] N. M. Chuong and T. D. Ke, "Generalized Cauchy problems involving nonlocal and impulsive conditions," Journal of Evolution Equations, vol. 12, no. 2, pp. 367-392, 2012.

[10] E. Hernández, M. Rabello, and H. R. Henríquez, "Existence of solutions for impulsive partial neutral functional differential equations," Journal of Mathematical Analysis and Applications, vol. 331, no. 2, pp. 1135-1158, 2007.

[11] E. Hernández, H. R. Henríquez, and M. A. McKibben, "Existence results for abstract impulsive second-order neutral functional differential equations," Nonlinear Analysis: Theory, Methods \& Applications, vol. 70, no. 7, pp. 2736-2751, 2009. 
[12] J. Hu and X. Liu, "Existence results of second-order impulsive neutral functional integrodifferential inclusions with unbounded delay in Banach spaces," Mathematical and Computer Modelling, vol. 49, no. 3-4, pp. 516-526, 2009.

[13] V. Kavitha, M. M. Arjunan, and C. Ravichandran, "Existence results for a second order impulsive neutral functional integrodifferential inclusions in Banach spaces with infinite delay," Journal of Nonlinear Science and its Applications, vol. 5, no. 5, pp. 321-333, 2012.

[14] V. Kavitha, M. M. Arjunan, and C. Ravichandran, "Existence results for non-densely defined impulsive neutral functional differential inclusions with state-dependent delay," International Journal of Nonlinear Science, vol. 13, no. 4, pp. 422-437, 2012.

[15] M. Lakrib, A. Oumansour, and K. Yadi, "Existence results for second order impulsive functional differential equations with infinite delay," The Australian Journal of Mathematical Analysis and Applications, vol. 9, no. 1, pp. 1-11, 2012.

[16] X. Liu and Y. Li, "Positive solutions for neumann boundary value problems of second-order impulsive differential equations in Banach spaces," Abstract and Applied Analysis, vol. 2012, Article ID 401923, 14 pages, 2012.

[17] V. Obukhovskii and J.-C. Yao, "On impulsive functional differential inclusions with Hille-Yosida operators in Banach spaces," Nonlinear Analysis: Theory, Methods \& Applications, vol. 73, no. 6, pp. 1715-1728, 2010.

[18] L. Byszewski, "Theorems about the existence and uniqueness of solutions of a semilinear evolution nonlocal Cauchy problem," Journal of Mathematical Analysis and Applications, vol. 162, no. 2, pp. 494-505, 1991.

[19] L. Byszewski and V. Lakshmikantham, "Theorem about the existence and uniqueness of a solution of a nonlocal abstract Cauchy problem in a Banach space," Applicable Analysis, vol. 40, no. 1, pp. 11-19, 1991.

[20] L. Byszewski, "Theorem about existence and uniqueness of continuous solution of nonlocal problem for nonlinear hyperbolic equation," Applicable Analysis, vol. 40, no. 2-3, pp. 173-180, 1991.

[21] L. Byszewski, "Uniqueness criterion for solution of abstract nonlocal Cauchy problem," Journal of Applied Mathematics and Stochastic Analysis, vol. 6, no. 1, pp. 49-54, 1993.

[22] L. Byszewski and H. Akca, "On a mild solution of a semilinear functional-differential evolution nonlocal problem," Journal of Applied Mathematics and Stochastic Analysis, vol. 10, no. 3, pp. 265-271, 1997.

[23] L. Byszewski and T. Winiarska, "An abstract nonlocal second order evolution problem," Opuscula Mathematica, vol. 32, no. 1, pp. 75-82, 2012.

[24] X. Fu, "On solutions of neutral nonlocal evolution equations with nondense domain," Journal of Mathematical Analysis and Applications, vol. 299, no. 2, pp. 392-410, 2004.

[25] E. Hernández M. and H. R. Henríquez, "Existence results for second order differential equations with nonlocal conditions in Banach spaces," Funkcialaj Ekvacioj, vol. 52, no. 1, pp. 113-137, 2009.

[26] D. Jackson, "Existence and uniqueness of solutions to semilinear nonlocal parabolic equations," Journal of Mathematical Analysis and Applications, vol. 172, no. 1, pp. 256-265, 1993.

[27] J. Wu, Theory and Applications of Partial Functional-Differential Equations, vol. 119, Springer, New York, NY, USA, 1996.
[28] E. P. Gatsori, L. Górniewicz, S. K. Ntouyas, and G. Y. Sficas, "Existence results for semilinear functional differential inclusions with infinite delay," Fixed Point Theory, vol. 6, no. 1, pp. 47-58, 2005.

[29] W. Arendt, C. J. K. Batty, M. Hieber, and F. Neubrander, VectorValued Laplace Transforms and Cauchy Problems, Birkhäuser, Basel, 2001.

[30] H. O. Fattorini, Second Order Linear Differential Equations in Banach Spaces, vol. 108 of North-Holland Mathematics Studies, North-Holland, Amsterdam, The Netherlands, 1985.

[31] M. Haase, The Functional Calculus for Sectorial Operators, vol. 169, Birkhäuser, Basel, Switzerland, 2006.

[32] S. I. Piskarev, "Evolution equations in Banach spaces. Theory of cosine operator functions," 2004, http://www.icmc.usp.br/andcarva/minicurso.pdf.

[33] V. V. Vasil and S. I. Piskarev, "Differential equations in Banach spaces. II: theory of cosine operator functions," Journal of Mathematical Sciences, vol. 122, no. 2, pp. 3055-3174, 2004.

[34] C. C. Travis and G. F. Webb, "Second order differential equations in Banach space," in Proceedings of the International Symposium on Nonlinear Equations in Abstract Spaces, pp. 331-361, Academic Press, New York, NY, USA, 1987.

[35] J. Kisyński, "On cosine operator functions and one-parameter groups of operators," Studia Mathematica, vol. 49, pp. 93-105, 1972.

[36] C. C. Travis and G. F. Webb, "Compactness, regularity, and uniform continuity properties of strongly continuous cosine families," Houston Journal of Mathematics, vol. 3, no. 4, pp. 555567, 1977.

[37] C. C. Travis and G. F. Webb, "Cosine families and abstract nonlinear second order differential equations," Acta Mathematica Academiae Scientiarum Hungaricae, vol. 32, no. 1-2, pp. 76-96, 1978.

[38] R. R. Akhmerov, M. I. Kamenski1̌, A. S. Potapov, A. E. Rodkina, and B. N. Sadovskil̆, Measures of Noncompactness and Condensing Operators, Birkhäuser, Basel, Switzerland, 1992.

[39] J. Banaś and K. Goebel, Measures of Noncompactness in Banach Spaces, vol. 60 of Lecture Notes in Pure and Applied Mathematics, Marcel Dekker, New York, NY, USA, 1980.

[40] H. R. Henríquez, “On non-exact controllable systems," International Journal of Control, vol. 42, no. 1, pp. 71-83, 1985.

[41] N. Dunford and J. T. Schwartz, Linear Operators-Part I: General Theory, John Wiley \& Sons, New York, NY, USA, 1988.

[42] H.-P. Heinz, "On the behaviour of measures of noncompactness with respect to differentiation and integration of vector-valued functions," Nonlinear Analysis: Theory, Methods \& Applications, vol. 7, no. 12, pp. 1351-1371, 1983. 


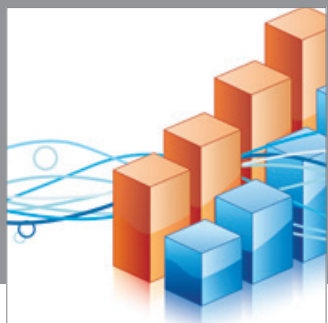

Advances in

Operations Research

mansans

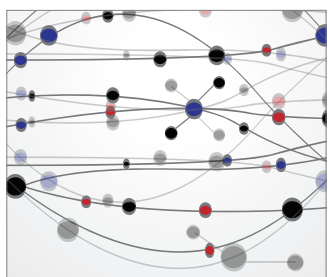

The Scientific World Journal
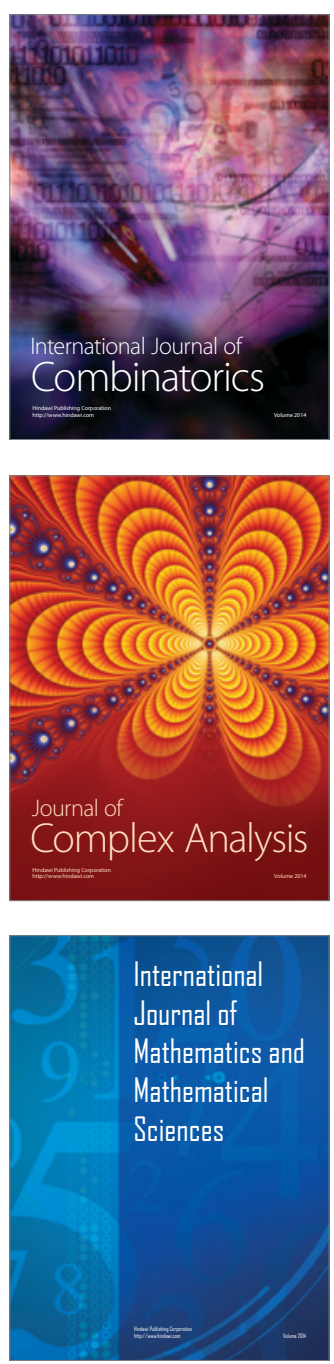
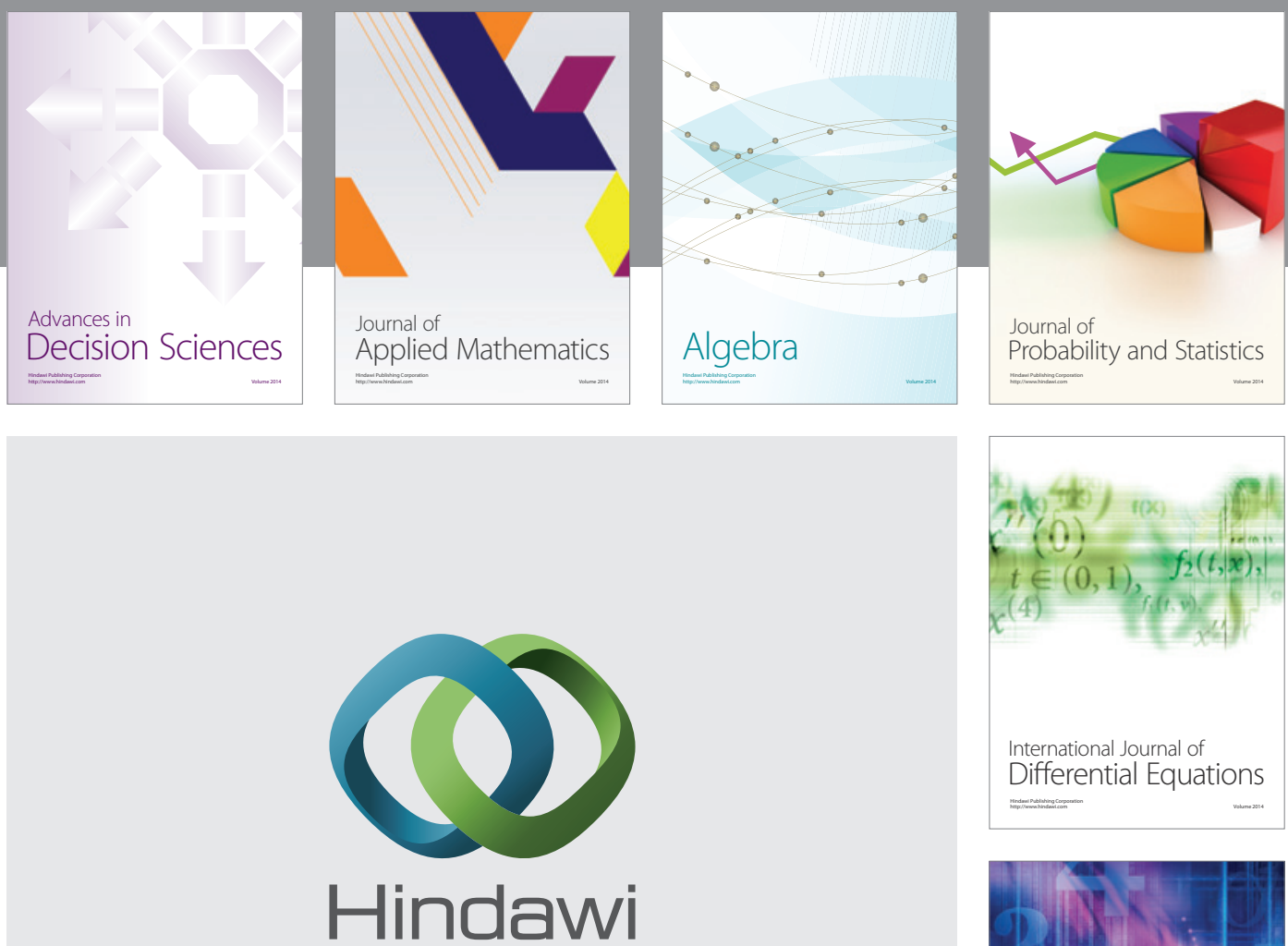

Submit your manuscripts at http://www.hindawi.com
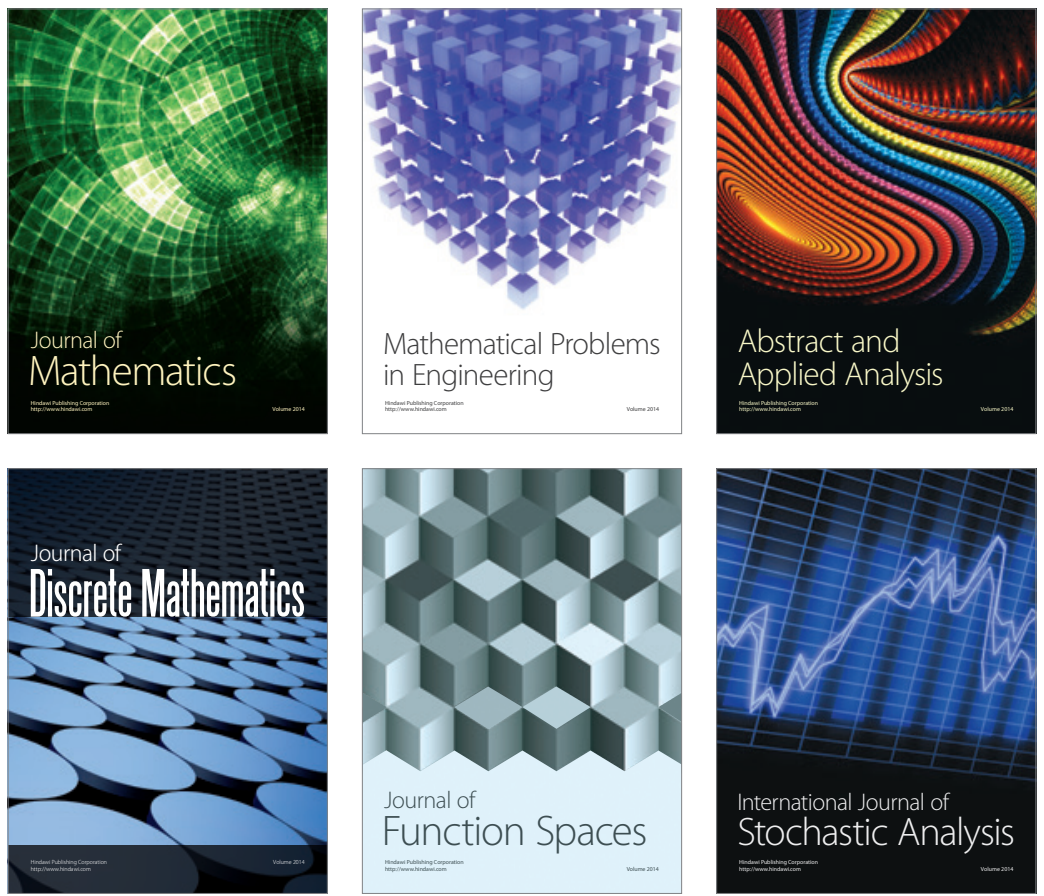

Journal of

Function Spaces

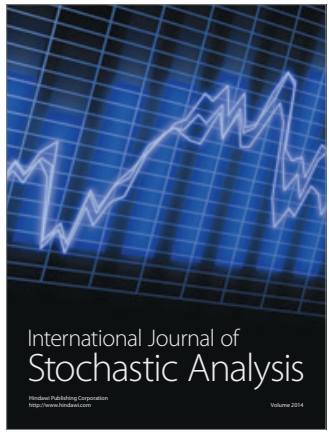

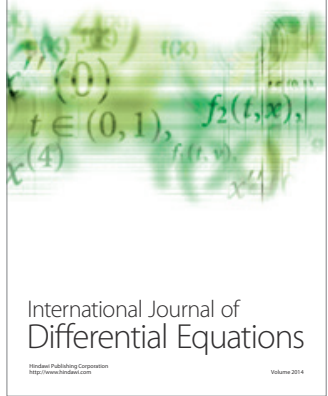
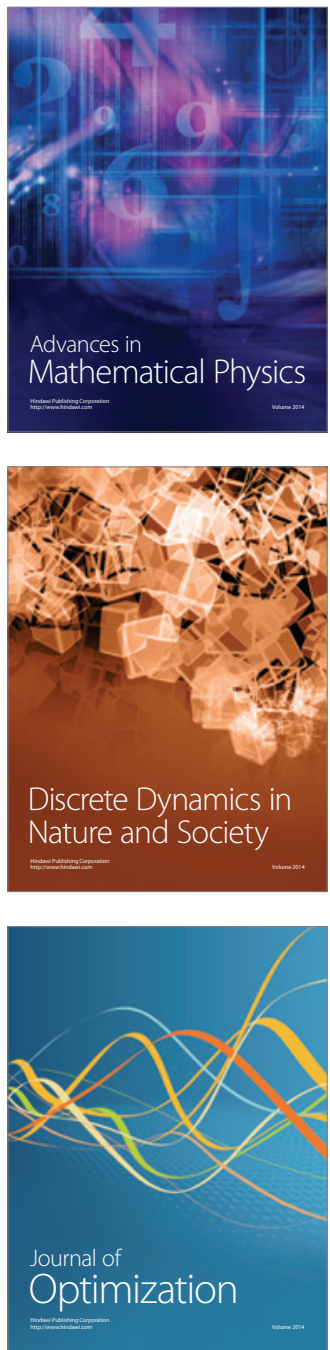